\title{
Microarray Analysis of the Juvenile Hormone Response in Larval Integument of the Silkworm, Bombyx mori
}

\author{
Daojun Cheng, ${ }^{1}$ Jian Peng, ${ }^{1}$ Meng Meng, ${ }^{1}$ Ling Wei, ${ }^{2}$ Lixia Kang, \\ Wenliang Qian, ${ }^{1}$ and Qingyou Xia ${ }^{1}$ \\ ${ }^{1}$ State Key Laboratory of Silkworm Genome Biology, Southwest University, No. 2, Tiansheng Road, Beibei District, \\ Chongqing 400715, China \\ ${ }^{2}$ School of Life Science, Southwest University, Chongqing 400715, China \\ Correspondence should be addressed to Qingyou Xia; xiaqy@swu.edu.cn
}

Received 22 November 2013; Revised 29 January 2014; Accepted 20 February 2014; Published 6 April 2014

Academic Editor: Prabhakara V. Choudary

Copyright (C) 2014 Daojun Cheng et al. This is an open access article distributed under the Creative Commons Attribution License, which permits unrestricted use, distribution, and reproduction in any medium, provided the original work is properly cited.

\begin{abstract}
Juvenile hormone $(\mathrm{JH})$ coordinates with 20-hydroxyecdysone (20E) to regulate larval growth and molting in insects. However, little is known about how this cooperative control is achieved during larval stages. Here, we induced silkworm superlarvae by applying the JH analogue (JHA) methoprene and used a microarray approach to survey the mRNA expression changes in response to JHA in the silkworm integument. We found that JHA application significantly increased the expression levels of most genes involved in basic metabolic processes and protein processing and decreased the expression of genes associated with oxidative phosphorylation in the integument. Several key genes involved in the pathways of insulin/insulin-like growth factor signaling (IIS) and 20E signaling were also upregulated after JHA application. Taken together, we suggest that JH may mediate the nutrient-dependent IIS pathway by regulating various metabolic pathways and further modulate $20 \mathrm{E}$ signaling.
\end{abstract}

\section{Introduction}

Juvenile hormone $(\mathrm{JH})$ is a sesquiterpenoid hormone that cooperates with 20-hydroxyecdysone (20E) to regulate many aspects of insect physiology, including growth, development, and reproduction $[1,2]$. In insects, JH is generally synthesized by the corpora allata and contributes to maintain larval growth $[2,3] .20 \mathrm{E}$ is transformed from ecdysone produced in the prothoracic glands and triggers larval molting and metamorphosis with the larval-pupal transition. Higher levels of $\mathrm{JH}$ during larval stages prevent metamorphosis, whereas lower levels or an absence of $\mathrm{JH}$ at the end of the larval or pupal stages allows 20E to promote metamorphosis [4-6]. JH also regulates female reproductive maturation in adult insects [6].

The mechanism mediating the JH response is a fascinating question. Recently, some studies have reported that $\mathrm{JH}$ can induce the transcription of a large number of genes in vivo or in vitro [7-14], and specific JH-response elements (JHREs) in some JH-regulated genes have been identified
[10]. However, little is known about the genomewide response to $\mathrm{JH}$ and the crosstalk between $\mathrm{JH}$ and $20 \mathrm{E}$ in insect larvae. Importantly, a transcription factor Krüppel homolog $1(K r-h 1)$ is transcriptionally regulated by $\mathrm{JH}$ and is involved in transducing the $\mathrm{JH}$ signal as a repressor of insect metamorphosis [15-19]. The binding motif of $\mathrm{Kr}-\mathrm{hl}$ in the promoter of $\mathrm{Kr}-\mathrm{h} 1$ target has been identified [20]. Therefore, $K r$ - $h l$ is regarded as an excellent indicator of JH sensitivity in insects [21].

Generally, insects feed and grow during their larval stages. $\mathrm{JH}$ is involved in maintaining metabolic homeostasis in insects, which is consistent with its function of keeping insects in the larval growth phase. For example, early evidence from the silkworm (Bombyx mori) showed that JH analogue (JHA) application impairs ATP production [22]. Recent reports found that $\mathrm{JH}$ regulates lipolysis and trehalose homeostasis in insects $[13,23,24]$. Interestingly, an evolutionarily conserved insulin/insulin-like growth factor signaling (IIS) pathway is also involved in mediating nutrition signaling during insect growth [25]. The IIS pathway participates in the nutritional regulation of $\mathrm{JH}$ biosynthesis in the yellow fever 
mosquito (Aedes aegypti) [26-28]. In addition, JH controls trehalose homeostasis to modulate starvation resistance by regulating the synthesis of insulin-like peptide 2 (ILP2) in the red flour beetle (Tribolium castaneum) [24]. However, our understanding of the metabolic pathways regulated by $\mathrm{JH}$ and their interconnections with the IIS pathway is limited.

The JH titer is high in the early stage of each larval instar whereas the $20 \mathrm{E}$ titer is elevated at the later stage. This fluctuation suggests an essential interplay between $\mathrm{JH}$ and 20E. To our current knowledge, some molecules, including Met, EcR, Broad complex, and Kr-hl, have been confirmed to orchestrate the crosstalk between $\mathrm{JH}$ and $20 \mathrm{E}$ during insect growth and development $[9,16,29-31]$. Intriguingly, several studies found that the IIS pathway regulates ecdysteroidogenesis [32-34]. These findings raise the possibility that $\mathrm{JH}$ may affect the $20 \mathrm{E}$ levels by modulating the activity of the nutrition-dependent IIS pathway.

The silkworm is an excellent model for studying crosstalk between $\mathrm{JH}$ and $20 \mathrm{E}$ because of the obvious boundary between feeding and molting during larval stages. There are several reports that focus on $\mathrm{JH}$ actions in the silkworm. For example, $\mathrm{JH}$ has been demonstrated to be a potent stimulator of the secretion of PTTH, a peptide that promotes ecdysteroidogenesis [35]. The overexpression of the juvenile hormone esterase gene that is involved in $\mathrm{JH}$ metabolism, the mutation of the Cyp15C1 gene that is involved in JH biosynthesis, or the direct downregulation of $\mathrm{JH}$ biosynthesis in the silkworm result in precocious metamorphosis and a reduction in larval molting times [36-38]. The application of the JHA methoprene at the beginning of the fourth larval instar induces perfectly the emergence of superlarvae with an additional larval instar [39]. JH is also involved in glycolysis and the immune response $[11,13,40]$. However, the genomewide response to $\mathrm{JH}$ and the genetic basis for the crosstalk between $\mathrm{JH}$ and other signaling pathways, such as the 20E signaling and IIS pathways, are still unclear in the silkworm.

To uncover the genomewide response to $\mathrm{JH}$ in vivo and to find new clues to the effect of $\mathrm{JH}$ on the signaling pathways related to larval growth and development, we investigated the genomewide changes of gene expression following JHA treatment. We measured gene expression in the integument (including muscle) of the silkworm using a silkworm microarray [41] and a silkworm superlarvae model induced by JHA application [39]. Our results show that JHA application increases the expression levels of genes involved in various metabolic processes and the IIS pathway in the silkworm.

\section{Materials and Methods}

2.1. Silkworm Strain. The silkworm strain Dazao (p50) with a characteristic of tetramolter (four molting times during larval stages) was used in our experiments. The larvae were reared on fresh mulberry leaves at $25^{\circ} \mathrm{C}$ under a 12-hour light/12hour dark photoperiod.

2.2. JHA Induction of Silkworm Superlarvae. We collected individual larvae one hour after the completion of the third larval molt and reared together to use for hormone treatment experiments. The JHA methoprene (Sigma, USA) was dissolved in acetone. According to previous report [35, 38, 39], doses of $10-30 \mu \mathrm{g}$ per larva were applied to newly molted fourth instar larvae along the dorsal midline of the larval thorax. Silkworm larvae treated with the same dose of pure acetone were used as a control.

2.3. Microarray Analysis. Larval integument (including muscle) is considered a peripheral tissue targeted by $\mathrm{JH}$ in insects [42]. To analyze the genomewide responses to JHA application in the silkworm integument, we separately isolated the integument from 20 larval individuals at different time points after JHA treatment, including 12 hours after treatment (Hat) with JHA or acetone (control), $24 \mathrm{Hat}, 36 \mathrm{Hat}$, and $48 \mathrm{Hat}$. Integument samples at each time point were immediately snap-frozen in liquid nitrogen and stored at $-80^{\circ} \mathrm{C}$. Three independent samples for each time point were isolated as biological replicates.

We further surveyed the changes of gene expression after JHA application using silkworm genomewide microarray, which has been widely used to profile gene expression in many studies [41, 43]. Total RNA extraction, microarray hybridization, raw data normalization, and microarray data analysis were performed as described in previous reports $[40,41]$. In brief, total RNA was extracted from the prepared sample using TRIzol reagent (Invitrogen, USA). Microarray hybridization and raw data normalization were performed by CapitalBio Corp. (China). Because of the high cost and the high reproducibility of microarray hybridization [41], we mixed equal amounts of total RNA from three biological replicates at each time point to create one sample for the hybridization experiment. cDNA for the microarray hybridization was prepared from each mixed RNA template, labeled with fluorescent dyes (Cy5 for the test samples and Cy3 for the control samples), and finally hybridized to the silkworm genome oligonucleotide microarray [41]. The raw signal intensity data for gene expression was extracted from the image obtained via scanning each hybridized array and normalized to the confirmed housekeeping genes.

For the microarray data analysis, we concluded that a gene was expressed in any sample at any time point if its expression signal intensity was greater than 400 units. The ratio of expression change for a gene in each time point after JHA application was evaluated by comparing with that in the control. This ration of expression change for a gene at each time point was further used to profile its time course expression change. Finally, a gene was considered as upor downregulated after JHA application if it displayed at least 2.0-fold change in expression level compared with the control. All these differentially expressed genes were defined as JHA-modulated genes. Hierarchical clustering of gene expression was performed using the Cluster 3.0 program [44]. To analyze parallel gene expression changes of the JHA-modulated genes across four selected time points, we performed a coexpression analysis using the GeneCluster 2.0 program [45]. All of the microarray data presented in this study have been deposited in the GEO database under accession number GSE53374. 
2.4. GO Annotation and KEGG Pathway Analysis. Using the online WEGO program [46], we functionally predicted the gene ontology (GO) terms of the JHA-modulated genes. In addition, we used the probe sequences of all up- and downregulated genes to BLAST search against the silkworm gene collection to obtain the probe-matched genes. The online KEGG pathway database (http://www.genome.jp/kegg/) and sequence similarities enabled these probe-matched genes to be mapped to different KEGG pathways using the KAAS (KEGG Automatic Annotation Server) tool [47].

2.5. Quantitative Real-Time RT-PCR Experiment. Using quantitative real-time RT-PCR experiments, we examined the changes in the mRNA expression of selected genes in the integument at 12, 24, 36, and 48 hours after JHA application. For an additional comparison, we evaluated the expression of the same gene collection in another JH-targeted tissue, the fat body, after JHA application. Because dissecting the fat body at the early stage of the fourth larval instar is difficult, we only dissected the fat body at 36 and 48 hours after JHA application. Three biological replicates were conducted. Sample collection, RNA extraction, and cDNA synthesis were performed according to previously described procedures [40].

Real-time RT-PCR was performed with the SYBR Premix Ex Taq system (TaKaRa Biotech, Japan). Each PCR reaction was conducted in a final volume of $20 \mu \mathrm{L}$ containing $70 \mathrm{ng}$ of cDNA $(2 \mu \mathrm{L})$, SYBR Premix Ex Taq $(10 \mu \mathrm{L})$, and $0.4 \mu \mathrm{M}$ primers. The PCR amplification conditions were set to the following: $95^{\circ} \mathrm{C}$ for $30 \mathrm{~s}$, followed by 40 cycles of $95^{\circ} \mathrm{C}$ for $30 \mathrm{~s}$ and $60^{\circ} \mathrm{C}$ for $30 \mathrm{~s}$. The mRNA expression levels of the selected genes were normalized to the control of translation initiation factor $4 \mathrm{~A}(e I F-4 A)$ gene. All primers used in this study are listed in Table S1. The Pearson correlation coefficient between microarray data and real time RT-PCR results was further calculated.

2.6. Prediction of Conserved Binding Sites of $K r-h 1$ on the Promoters of JHA-Induced Genes. We fetched the sequences from the approximately $3 \mathrm{~kb}$ upstream untranslated region (UTR) of translation initiation site of genes that their mRNA expressions were upregulated with at least 1.5 -fold at 12 hours after JHA application. These sequences were used to search the conserved recognition and binding motif of transcription factor $\mathrm{Kr}-\mathrm{hl}$, a key transducer of $\mathrm{JH}$ signal, by the online MatInspector program (http://www.genomatix.de/) [20, 48].

\section{Results}

3.1. Induction of Silkworm Superlarvae by JHA Application. To determine the gene expression changes in silkworm in response to $\mathrm{JH}$, we used the JHA methoprene to induce silkworm superlarvae according to previous reports $[38,39]$. After several doses of JHA were applied topically to newly molted fourth instar larvae, we found that 10 or $20 \mu \mathrm{g}$ of JHA per larva induced silkworm superlarvae with a high probability of $93.3 \%$. However, a dose of $30 \mu \mathrm{g}$ per larva resulted in approximately 50\% larval death before the fourth molting (Figure 1(a)).

Therefore, we applied $20 \mu \mathrm{g}$ of JHA per larva to hundreds of silkworm larvae for further analysis. Compared with pure acetone treatment as control, the JHA application initiated premature fourth molting and led to an additional fifth molting, with the consequence of becoming superlarvae (Figures 1(b) and 1(c)). Although the developmental duration of the fourth and fifth larval instars of silkworm superlarvae was shortened owing to an additional sixth larval instar, the entire larval stage was extended by approximately six days, and the average weight per individual was elevated before wandering (Figure 1(d)). Furthermore, quantitative RT-PCR examination showed that in silkworm superlarvae, the JH-regulated marker gene $K r-h 1$ displayed a significantly upregulated expression in the integument at 12, 24, 36, and 48 Hat (Figure 1(e)) and in the fat body at 36 and 48 Hat.

\subsection{JHA Application Induced a Global Alteration of Gene} Expression. Based on the model of JHA-induced silkworm superlarvae, we used a genomewide silkworm microarray to analyze the global gene expression pattern in the integument following JHA application. As shown in Table S2 and Figure S1, a total of 8,670 genes were expressed in the integuments with both treatments from JHA and pure acetone. Scatter plot analysis demonstrated that the expression levels of many genes were altered by JHA application (Figure 2(a)). Intriguingly, 2,143 genes exhibited an expression change of at least 2.0-fold after JHA application (Table S3). For convenience in the following description, we defined these genes as JHA-modulated genes. In detail, at 12 Hat, 49 and 87 genes were up- and downregulated, respectively. At $24 \mathrm{Hat}$, 69 and 74 genes were up- and downregulated, respectively. At 36 and 48 Hat, respectively, 450 and 857 genes were upregulated, while 165 and 956 genes were downregulated. Further real-time RT-PCR experiments confirmed that most of the examined genes, whose expressions were deregulated after JHA application, showed good correlations (Pearson correlation coefficient, $r>0.7$ ) between microarray data and RT-PCR results (Table S4).

GO annotation showed that JHA-modulated genes could be functionally classified into 32 categories (Figure 2(b)). The number of JH-modulated genes belonging to the categories of catalytic activity and metabolic process was the largest. In addition, KEGG analysis revealed that JHA-modulated genes were mainly related to metabolism, genetic information processing, environmental information processing, cellular processes, and organismal systems (Figure 2(c); Table S5). Remarkably, after a redundancy subtraction for probes, we noted that JHA-modulated genes involved in basic metabolic processes have the largest number (i.e., 302).

GeneCluster-based coexpression analysis revealed that the expression changes of most JHA-modulated genes in the integument exhibited a similar time course dependence. As shown in Figure 2(d) and Table S6, the dynamic expression of JHA-modulated genes could be grouped into eight clusters. JHA-modulated genes from the C0, C1, C2, and C6 clusters were upregulated at 48 Hat. The C3 and C4 clusters showed 


\begin{tabular}{cccccc}
\hline Dose & $\begin{array}{c}\text { Injected } \\
\text { number }\end{array}$ & $\begin{array}{c}\text { Fourth } \\
\text { molting }\end{array}$ & $\begin{array}{c}\text { Death before } \\
\text { fourth molting }\end{array}$ & $\begin{array}{c}\text { Fifth } \\
\text { molting }\end{array}$ & $\begin{array}{c}\text { Percent of } \\
\text { superlarvae }\end{array}$ \\
\hline Acetone & 30 & 30 & 0 & 0 & 0 \\
$10 \mu \mathrm{g}$ & 30 & 30 & 0 & 28 & 93.3 \\
$20 \mu \mathrm{g}$ & 30 & 30 & 0 & 28 & 93.3 \\
$30 \mu \mathrm{g}$ & 30 & 16 & 14 & 16 & 53.3 \\
\hline
\end{tabular}

(a)

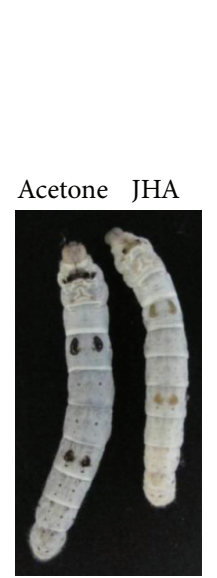

(c)

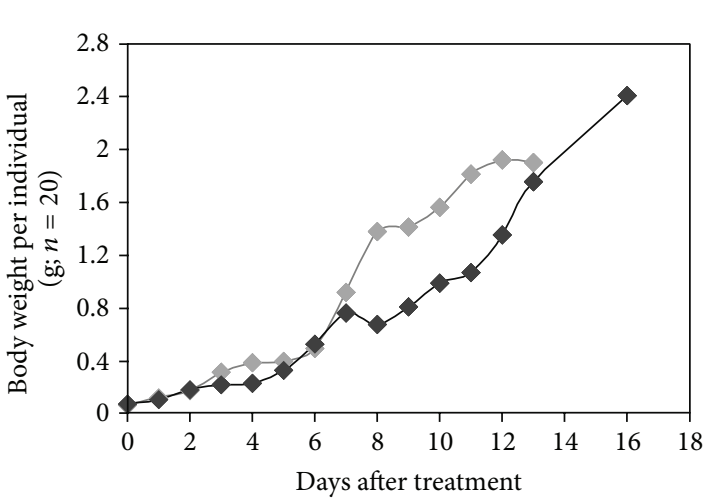

$-\rightarrow$ Acetone

$\rightarrow$ JHA

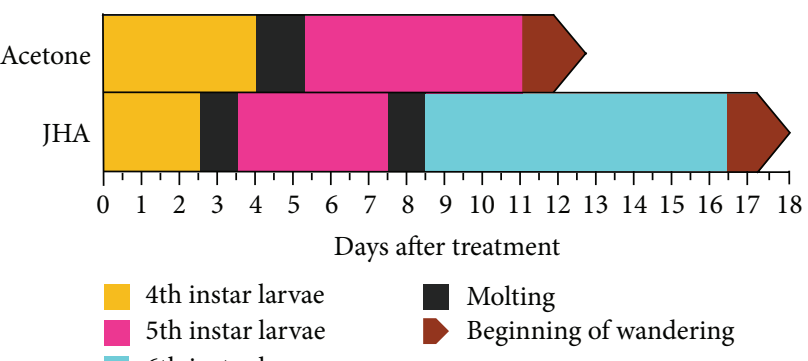

(b)

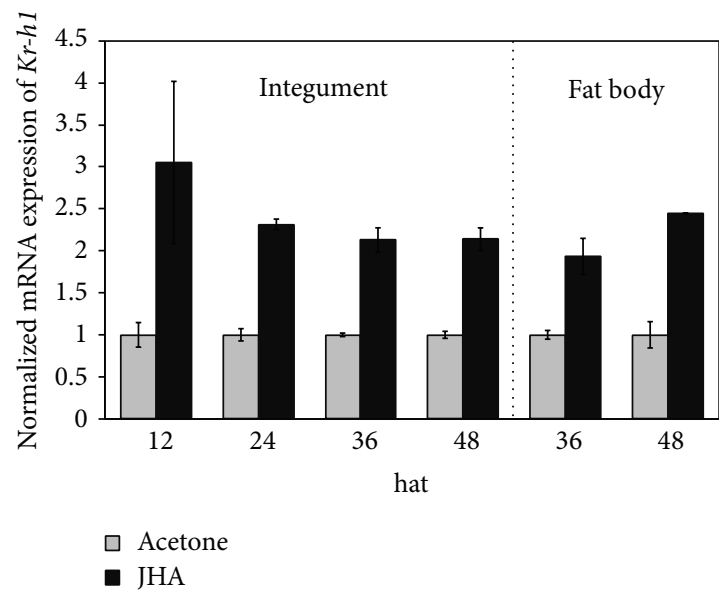

(e)

FIGURE 1: Induction of silkworm superlarvae by JHA application. Silkworm superlarvae were induced by applying the JH analogue (JHA) methoprene to newly molted silkworm larvae at the fourth instar. Silkworm larvae treated with pure acetone were used as a control. (a) Dose-dependent effects of JHA application on silkworm larval molting and survival. (b) JHA application at a dose of $20 \mu \mathrm{g}$ per larva results in precocious larval molting and induces silkworm superlarvae. (c) Comparison of normal larva with pure acetone treatment (left) and larva with precocious onset of the fourth larval molting at 48 hours after JHA application. The developmental time point is at approximately 60-72 hours after treatment. (d) JHA application changes the body weight of silkworm larvae. (e) The mRNA expression of $\mathrm{Kr}$ - $\mathrm{hl}$, a JH-responsive gene, is increased after JHA application. Hat: hours after treatment.

an upregulation at 12 and 36 Hat as well as a downregulation at 24 and 48 Hat. C5 was upregulated at 36 Hat and then downregulated at $48 \mathrm{Hat}$. $\mathrm{C} 7$ showed a significant downregulation from 12 to 48 Hat.

We used MatInspector program to search the binding motifs of transcription factors in the upstream UTR regions of the translation start sites of 157 genes whose expression showed upregulation with at least 1.5 -fold at 12 Hat after JHA application. Our results found that the conserved binding motif of $\mathrm{Kr}$-hl, which is a key transcription factor for mediating JH signal, was present within $3 \mathrm{~kb}$ upstream UTR regions of 95 of the $157 \mathrm{JHA}$-induced genes at 12 Hat (Table S7). Importantly, as shown in Figure 2(e), the Kr-h1 binding motifs of 67 JHA-induced genes revealed the highest core similarity (Core sim) of 1 with the binding motif of Kr-h1 [20], which all were comprised of a consensus sequence GGGT. This indicates that these JHA-induced genes may be the direct targets of silkworm Kr-h1.

3.3. JHA Application Facilitates Basic Metabolic Processes. Because the number of JHA-modulated genes involved in metabolic pathways was the highest and the main function of $\mathrm{JH}$ is to maintain insect larval growth, we further investigated JHA-modulated genes related to metabolic pathways. Our results from the KEGG analysis showed that 99 JHA-modulated genes were involved in carbohydrate metabolism (Figure 3; Table S8), with 74 genes that were upregulated in the integument after JHA application and that could be classified as members of the $\mathrm{C} 0, \mathrm{C} 1, \mathrm{C} 2$, C5, or C6 clusters (Table S6). Interestingly, we observed that JHA-modulated genes involved in three pathways of carbohydrate metabolism were all upregulated, including ten genes for the pentose phosphate pathway (e.g., ribosephosphate pyrophosphokinase, 6-phosphogluconate dehydrogenase, 6-phosphogluconolactonase, phosphoglucomutase, transketolase, fructose-1,6-bisphosphatase I, fructosebisphosphate aldolase, and glucose-6-phosphate isomerase), five genes involved in propanoate metabolism (i.e., 4aminobutyrate aminotransferase, aldehyde dehydrogenase, aldehyde dehydrogenase family 7 member A1, acetyl-CoA Cacetyltransferase, and enoyl-CoA hydratase), and five genes involved in butanoate metabolism (i.e., 3-oxoacid CoAtransferase, 4-aminobutyrate aminotransferase, enoyl-CoA 


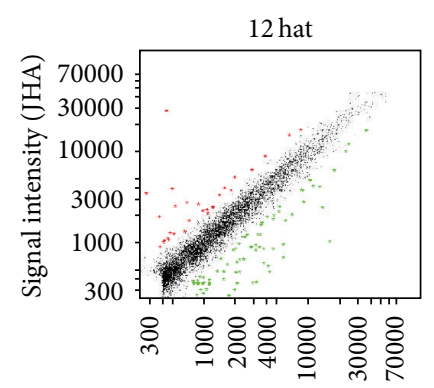

Signal intensity (acetone)

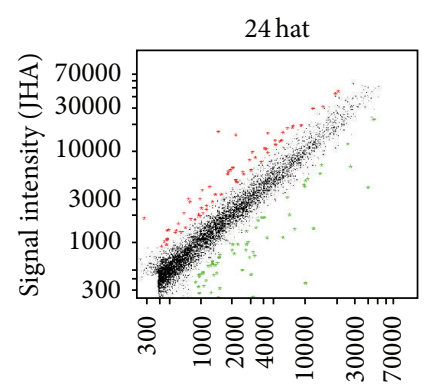

Signal intensity (acetone)

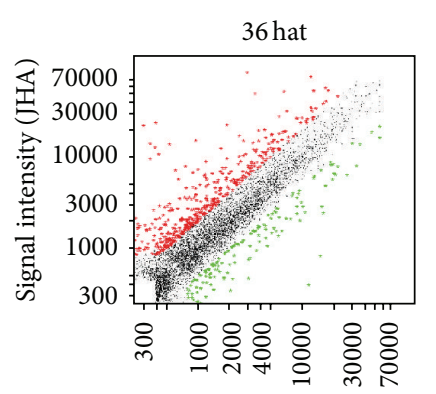

Signal intensity (acetone)

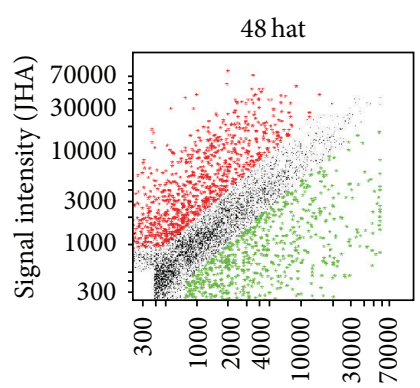

Signal intensity (acetone)

(a)

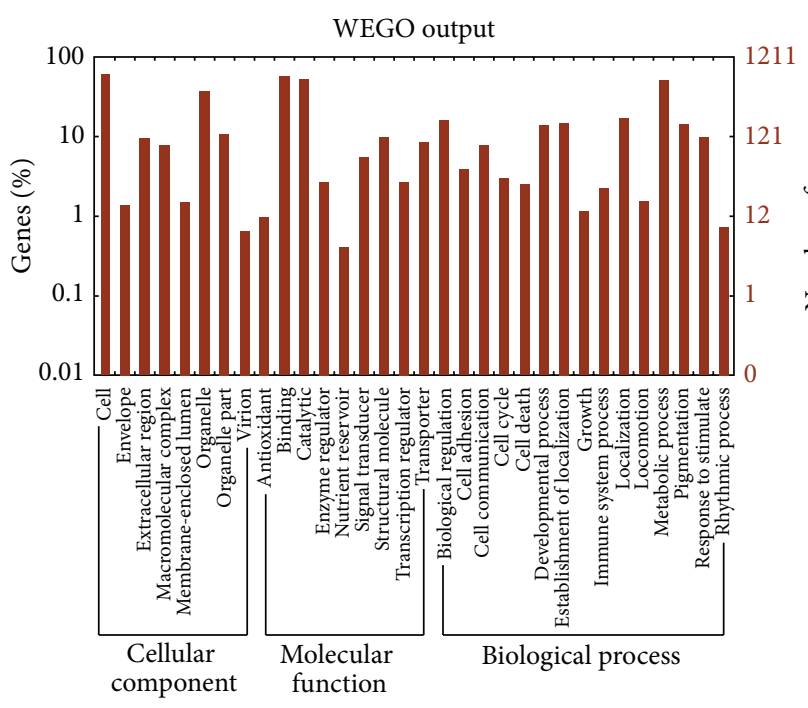

(b)

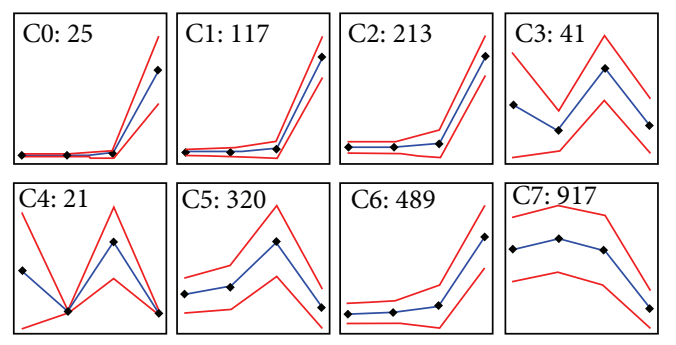

(d)

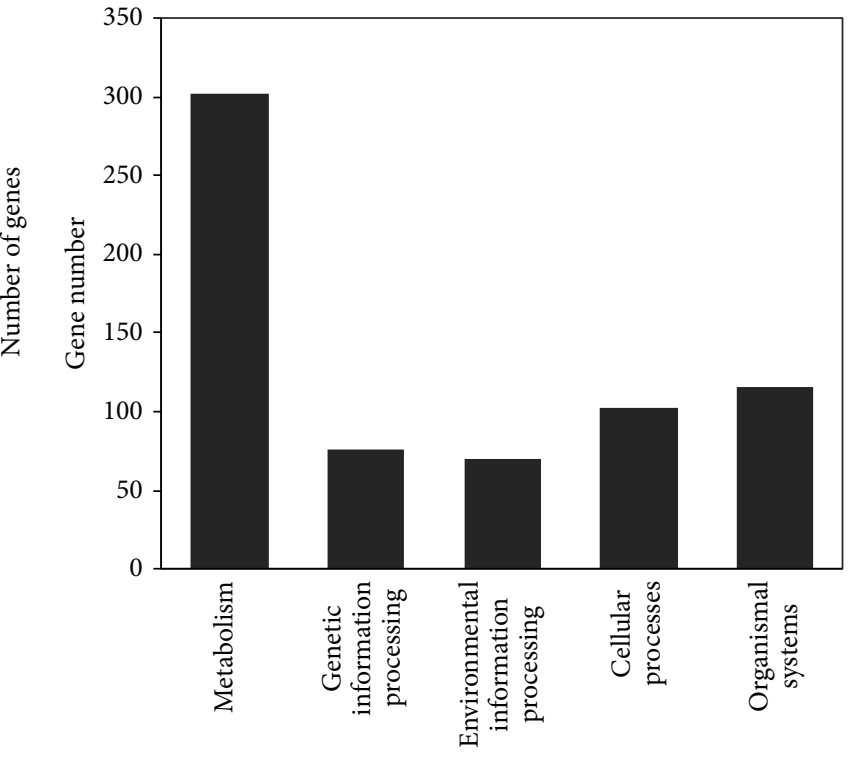

(c)

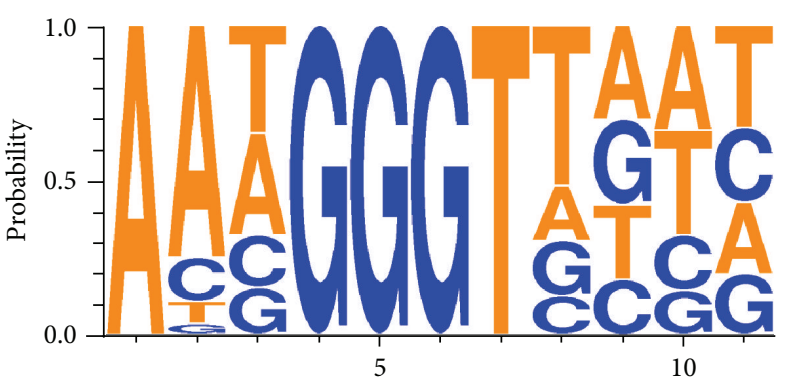

(e)

FIGURE 2: Genomewide alteration of gene expression after JHA application. Genomewide microarray analysis revealed that gene expressions in the integument and fat body were altered after JHA application. (a) Scatter plot of genomewide changes in gene expression. Hat: hours after treatment. (b) Gene ontology (GO) annotation of JHA-modulated genes with an at least 2.0-fold change in expression level after JHA application. All JHA-modulated genes are listed in Table S3 available online at http://dx.doi.org/10.1155/2014/426025. (c) JHA-modulated genes can be functionally classified into different KEGG pathways. For details see Tables S5 and S8. (d) Time course-dependant coexpression of JHA-modulated genes after JHA application in the integument. The digits denote the number of JHA-modulated genes in each cluster. The JHA-modulated genes in each cluster are listed in Table S6. (e) Multiple alignments of conserved binding motifs of Kr-h1 within the upstream UTR regions of JHA-induced genes.

hydratase, hydroxymethylglutaryl-CoA lyase, and acetylCoA C-acetyltransferase). Similarly, most of the genes implicated in other several carbohydrate metabolism pathways (such as glycolysis, pentose and glucuronate interconversions, galactose metabolism, starch and sucrose metabolism, amino sugar and nucleotide sugar metabolism, pyruvate metabolism, and glyoxylate and dicarboxylate metabolism) were also upregulated by JH application.

The KEGG analysis showed that 47 JHA-modulated genes are involved in energy metabolism (Figure 4; Table 

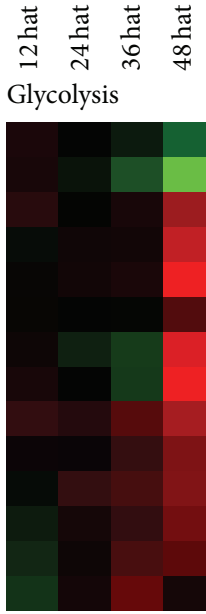

sw06503 sw10994 sw04448 sw09712 sw00132 sw19274 sw03637 sw19777 sw06691 sw17679 sw21062 sw17859 sw22892 sw18911

TCA cycle

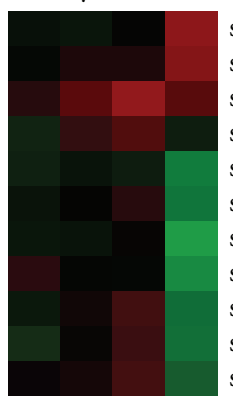

sw06088 sw06329 sw01281 sw14808 sw08124 sw01836 sw13400 sw10755 sw05999 sw10937 sw20350

Pentose phosphate pathway

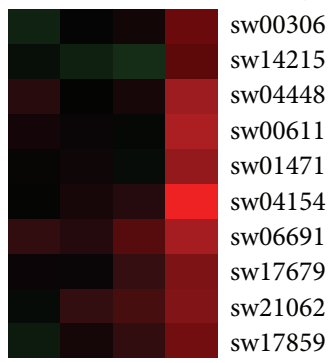

Fructose and mannose metabolism

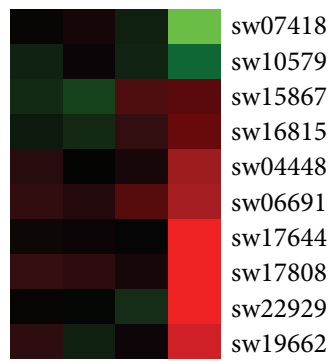

$\begin{array}{lllllll}-3 & -2 & -1 & 0 & 1 & 2 & 3\end{array}$

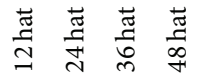

Galactose metabolism

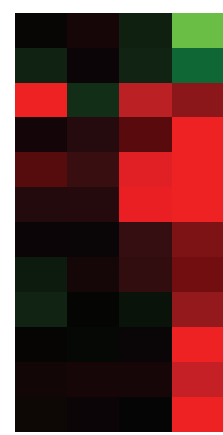

sw07418 sw10579 sw03428 sw05025 sw07028 sw09747 sw17679 sw17859 sw11414 sw08156 sw12759 sw17644

Pentose and glucuronate interconversions

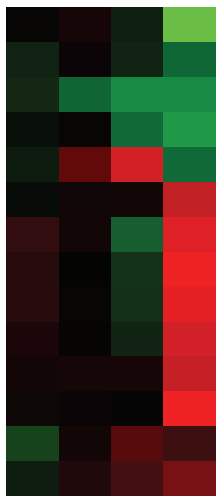

sw07418 sw10579 sw06973 sw18729 sw13973 sw09712 sw05953 sw07985 sw03726 sw21098 sw12759 sw17644 sw19163 sw22710

Starch and sucrose metabolism

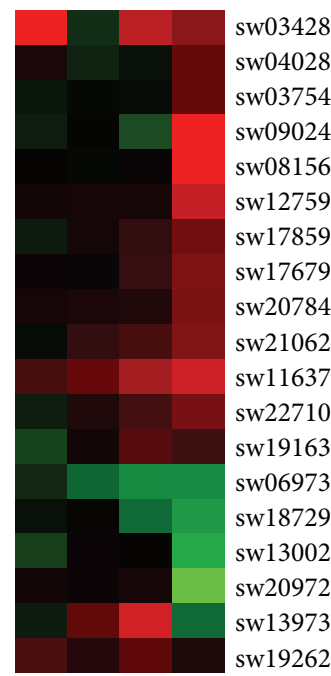

$\begin{array}{lllllll}-3 & -2 & -1 & 0 & 1 & 2 & 3\end{array}$

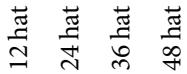

Propanoate metabolism

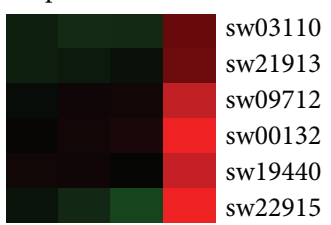

Amino sugar and nucleotide sugar metabolism

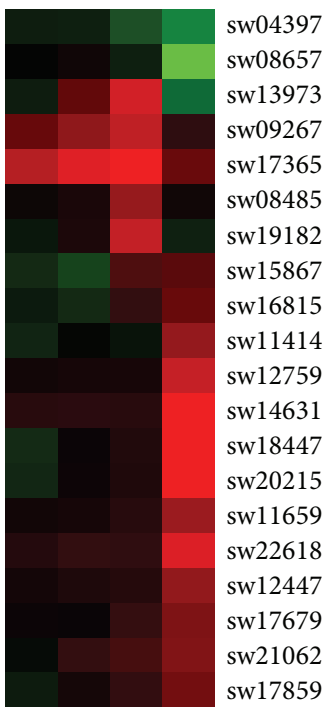

Glyoxylate and dicarboxylate metabolism

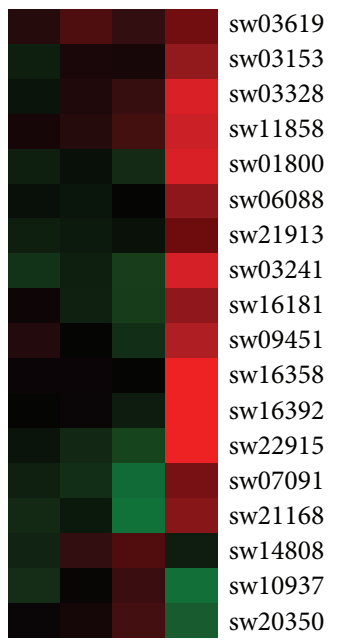

$\begin{array}{lllllll}-3 & -2 & -1 & 0 & 1 & 2 & 3\end{array}$

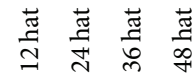

Pyruvate metabolism

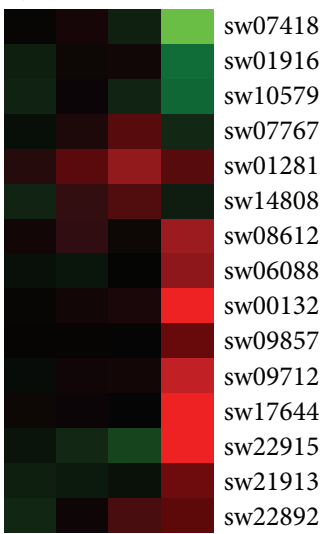

Butanoate metabolism

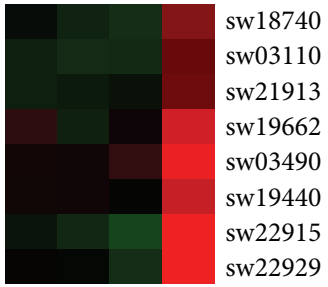

Inositol phosphate metabolism

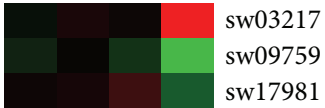

Ascorbate and aldarate metabolism

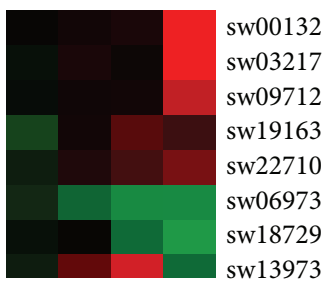

$\begin{array}{lllllll}-3 & -2 & -1 & 0 & 1 & 2 & 3\end{array}$

FIGURE 3: JHA-modulated pathways related to carbohydrate metabolism. Hierarchical clustering of JHA-modulated genes involved in 14 pathways related to carbohydrate metabolism. The probe ID, KEGG ID, and expression intensity of each JHA-modulated gene are listed in Tables S5 and S8. Hat: hours after treatment. 
S8). In addition, 25 JHA-modulated genes were upregulated, with most (18) of them belonging to cluster C6, which is upregulated at 48 Hat. In detail, more than half of the genes involved in each of the four pathways related to energy metabolism, including methane metabolism, nitrogen metabolism, carbon fixation in photosynthetic organisms, and carbon fixation pathways in prokaryotes, were upregulated. Intriguingly, we found that all $15 \mathrm{JH}$-modulated genes involved in the program of oxidative phosphorylation (OXPHOS) were downregulated, including 13 types of $\mathrm{V}$ type $\mathrm{H}^{+}$-transporting ATPase subunits and two succinate dehydrogenase (ubiquinone) subunits.

There are 58 enzyme-encoding genes involved in 14 pathways related to lipid metabolism that were modulated by JHA (Figure 4; Table S8). Of these genes, 38 were upregulated. All 11 genes catalyzing the biosynthesis of fatty acids and unsaturated fatty acids were upregulated after JHA induction, such as fatty acid synthase, 3-oxoacyl-[acyl-carrier protein] reductase, very-long-chain 3-oxoacyl-CoA reductase, stearoyl-CoA desaturase (delta-9 desaturase), and acyl-CoA oxidase. Similarly, among 18 genes involved in the elongation and metabolism of fatty acids, 14 genes were upregulated after JHA application, including acetyl-CoA acyltransferase, palmitoyl-protein thioesterase, aldehyde dehydrogenase, long-chain acyl-CoA synthetase, very-long-chain acyl-CoA dehydrogenase, and enoyl-CoA hydratase. Furthermore, four genes participating in the synthesis and degradation of ketone bodies were also induced by JHA.

Among the 37 JHA-modulated genes involved in glycogen biosynthesis and metabolism, 34 were upregulated after JHA application (Figure 4; Table S8). Notably, all JHAmodulated genes involved in the biosynthesis of several types of glycans (e.g., N-glycan, mucin-type O-glycan, glycosaminoglycan, and lipopolysaccharide) were upregulated. In addition, JH-modulated genes for glycosaminoglycan degradation all increased their expression after JHA application, including alpha-1,2-mannosyltransferase, arylsulfatase B, hexosaminidase, xylosylprotein 4-beta-galactosyltransferase, mannosyl-oligosaccharide alpha-1,2-mannosidase, betagalactosidase, and oligosaccharyltransferase complex subunit beta.

Our KEGG analysis indicated that 117 JHA-modulated genes were involved in 20 pathways related to amino acid metabolism, with 96 being upregulated after JHA application (Figure 5; Table S8). These pathways involved a large number of JHA-modulated enzymes mainly included the metabolism of glycine, serine, and threonine, the degradation of valine, leucine, and isoleucine, the metabolism of arginine and proline, and the metabolism of tryptophan. The number of JHA-modulated genes for these four pathways was 23, 23,19 , and 17 , respectively. In addition, 12 enzyme-coding genes involved in beta-alanine metabolism were all upregulated after JHA application, including dihydropyrimidine dehydrogenase, 4-aminobutyrate aminotransferase, aldehyde dehydrogenase, spermine synthase, beta-ureidopropionase, enoyl-CoA hydratase, and aldehyde dehydrogenase family 7 member A1.

We also noted that 26 JHA-modulated genes were responsible for catalyzing the metabolism of two types of nucleotides: purine and pyrimidine (Figure 5; Table S8). Importantly, most (24) of these genes were upregulated after JHA application, including 5 '-nucleotidase, adenylate kinase, polyribonucleotide nucleotidyltransferase, urate oxidase, pyruvate kinase, ribose-phosphate pyrophosphokinase, phosphoglucomutase, dihydropyrimidine dehydrogenase, adenylosuccinate lyase, phosphoribosylformylglycinamidine synthase, phosphoribosylaminoimidazole carboxylase, beta-ureidopropionase, purine-nucleoside phosphorylase, and ribonucleoside-diphosphate reductase subunit.

3.4. JHA Application Promoted Protein Synthesis and Degradation. Given that that $\mathrm{JH}$ is a key regulator for controlling insect growth and that JHA application enhanced a variety of basic metabolic processes, we evaluated the JHA-induced response of factors involved in protein processing pathways including synthesis and degradation. The KEGG analysis revealed that 97 JHA-modulated genes were involved in protein synthesis and degradation, with 79 being upregulated after JHA application (Figure 6; Table S8). Among the $41 \mathrm{JH}$-modulated genes involved in protein processing in the endoplasmic reticulum, 34 were upregulated following JHA application, including transitional endoplasmic reticulum ATPase, protein disulfide-isomerase, mannosyloligosaccharide alpha-1,2-mannosidase, protein transport protein SEC6, and translocation and translocation-associated proteins.

The expression of eight JHA-modulated genes related to protein export were increased after JHA application (Figure 6; Table S8), including protein transport protein SEC61 subunit alpha, protein transport protein SEC61 subunit gamma, translocation protein SEC63, signal peptidase complex subunit 2, signal recognition particle subunit SRP54, and signal recognition particle receptor subunit alpha.

We noted that the expression levels of most JHAmodulated genes associated with protein degradation were also upregulated after JHA application (Figure 6; Table S8), including five genes related to ubiquitin-mediated proteolysis (i.e., E3 ubiquitin-protein ligase synoviolin, WW domaincontaining E3 ubiquitin protein ligase 1, E3 ubiquitin-protein ligase HERC2, DNA damage-binding protein 1, and Fbox and WD-40 domain protein), two genes involved in proteasome formation (i.e., $26 \mathrm{~S}$ proteasome regulatory subunits $\mathrm{N} 1$ and N2), 22 genes associated with the lysosome (including four cathepsin family members, hexosaminidase, arylsulfatase B, glucosylceramidase, and beta-galactosidase), and 13 genes associated with the peroxisome (including catalase, phytanoyl-CoA hydroxylase, hydroxymethylglutarylCoA lyase, (S)-2-hydroxy-acid oxidase, fatty acyl-CoA reductase, isocitrate dehydrogenase, and superoxide dismutase).

3.5. The IIS Pathway Was Increased by JHA Application. Intriguingly, our microarray data showed that the expression level of the insulin receptor gene InR (probe ID: sw20525) was increased in the integument following JHA 


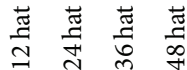

Oxidative phosphorylation

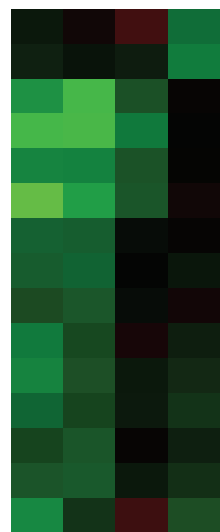

sw05999
sw08124
sw09511
sw09128
sw12174
sw04026
sw14793
sw07674
sw14397
sw00795
sw14354
sw09888
sw21938
sw13277
sw22916

Carbon fixation in photosynthetic organisms

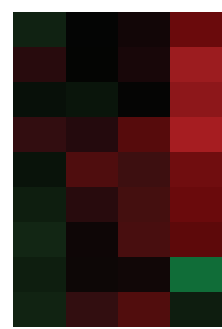

sw00306 sw04448 sw06088 sw06691 sw10674 sw14438 sw22892 sw01916 sw14808

Carbon fixation pathways in prokaryotes

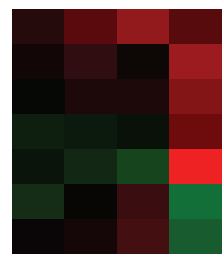

sw01281 sw08612 sw06329 sw21913 sw22915 sw10937 sw20350

Nitrogen metabolism

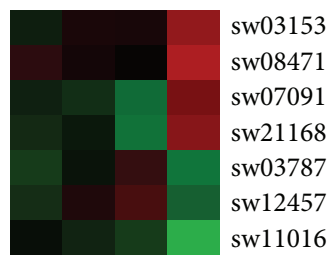

$\begin{array}{lllllll}-3 & -2 & -1 & 0 & 1 & 2 & 3\end{array}$

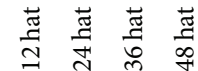

Methane metabolism

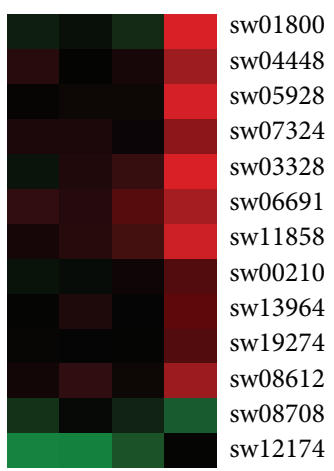

Fatty acid biosynthesis

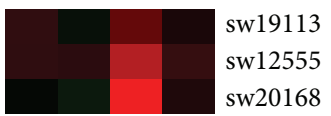

Fatty acid elongation

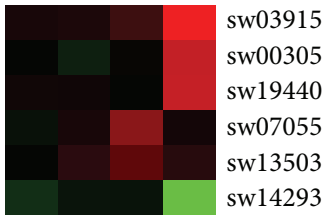

Fatty acid metabolism

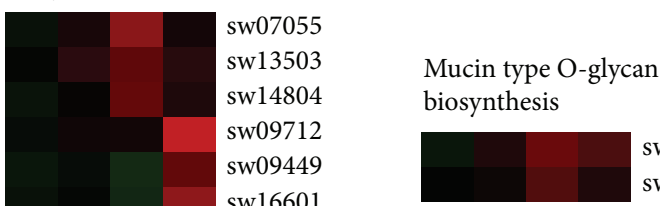

sw14162 sw20932

Glycosaminoglycan biosynthesis
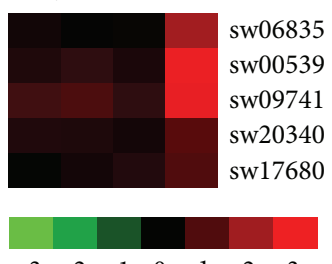

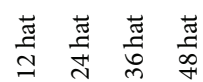

Glycosphingolipid biosynthesis

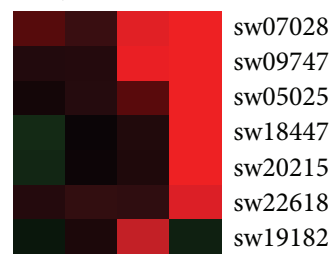

Glycosaminoglycan degradation

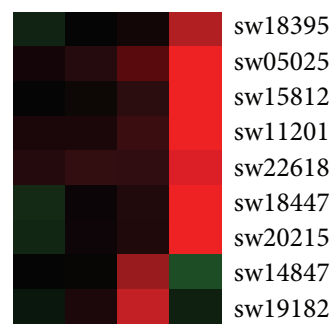

Other glycan degradation

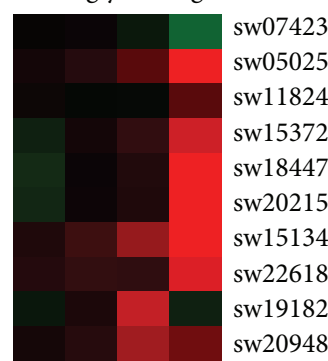

$\begin{array}{lllllll}-3 & -2 & -1 & 0 & 1 & 2 & 3\end{array}$

FIGURE 4: JHA-modulated pathways related to the metabolism of energy, lipids, or glycans. Cluster analysis of JHA-modulated genes involved in five pathways related to energy metabolism, five pathways related to lipid metabolism, and six pathways related to glycan metabolism. The probe ID, KEGG ID, and expression intensity of each JHA-modulated gene are listed in Tables S5 and S8. Hat: hours after treatment.

application (Figure 7(a); Table S8), which was also confirmed in the integument and fat body using quantitative real-time RT-PCR (Figure 7(b)). We further examined the expression of other JHA-modulated genes related to the IIS pathway in our microarray data. The result showed that
21 of 29 JH-modulated genes involved in the IIS pathway were upregulated after JHA application, including two genes for insulin secretion (i.e., solute carrier family 2 and sodium/potassium-transporting ATPase subunit beta), five genes for insulin signaling (receptor-type tyrosine-protein 


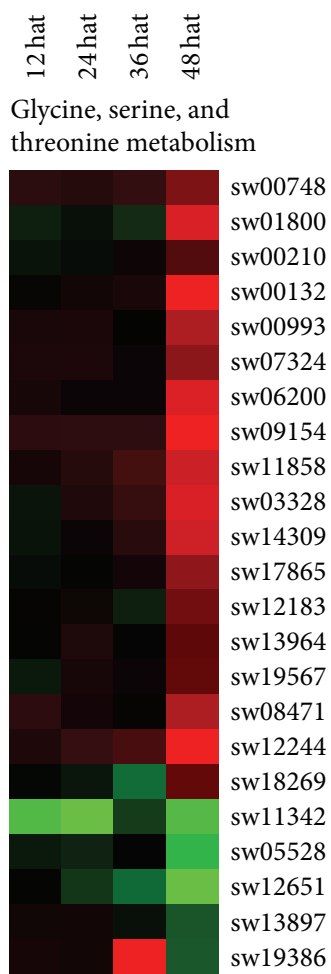

Valine, leucine, and isoleucine degradation

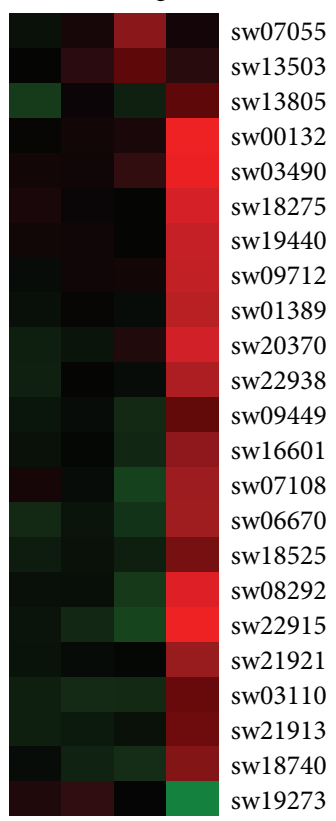

$\begin{array}{lllllll}-3 & -2 & -1 & 0 & 1 & 2 & 3\end{array}$

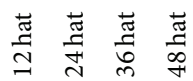

Arginine and proline metabolism

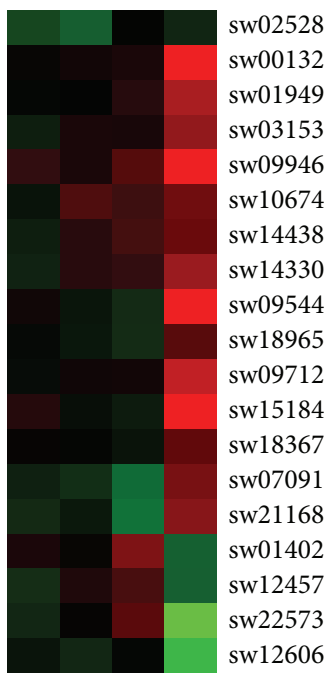

Glutathione metabolism

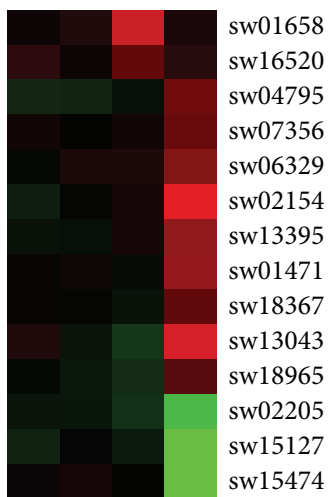

Lysine degradation

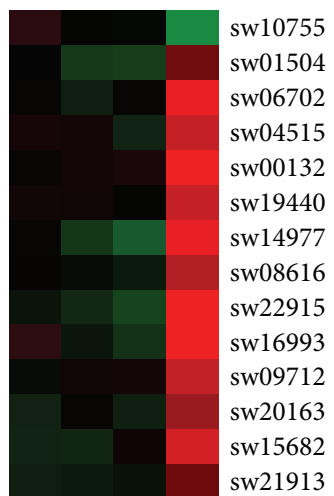

$\begin{array}{lllllll}-3 & -2 & -1 & 0 & 1 & 2 & 3\end{array}$

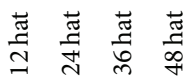

Tryptophan

metabolism

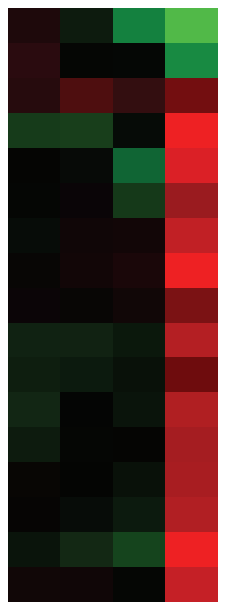

sw02887 sw10755 sw03619 sw00689 sw09629 sw22291 sw09712 sw00132 sw22405 sw12156 sw21913 sw10126 sw18242 sw22810 sw08616 sw22915 sw19440

Alanine, aspartate, and glutamate metabolism

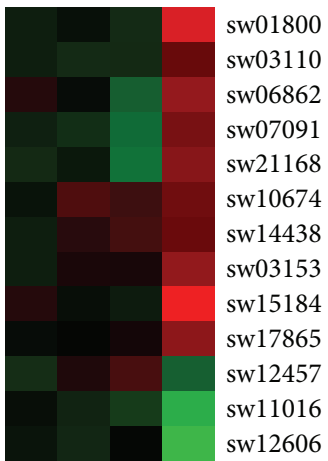

Beta-alanine metabolism
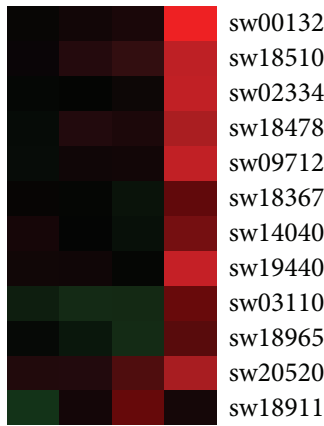

$\begin{array}{lllllll}-3 & -2 & -1 & 0 & 1 & 2 & 3\end{array}$

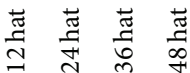

Purine

metabolism

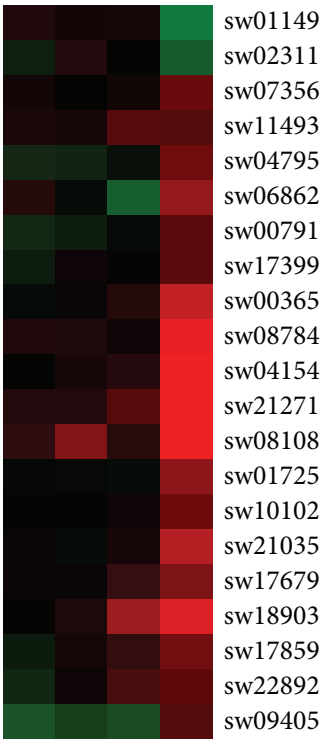

Pyrimidine metabolism

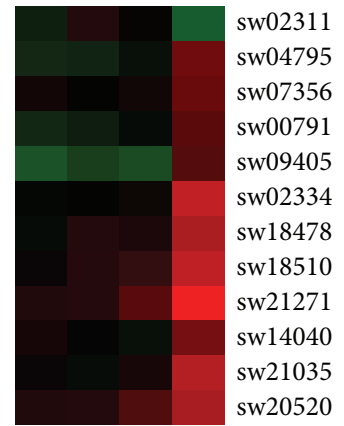

$\begin{array}{lllllll}-3 & -2 & -1 & 0 & 1 & 2 & 3\end{array}$

FIGURE 5: JHA-modulated pathways involved in amino acid and nucleotide metabolisms. Hierarchical clustering of JHA-modulated genes involved in eight pathways related to amino acid metabolism and two pathways related to nucleotide metabolism. The probe ID, KEGG ID, and expression intensity of each JHA-modulated gene are listed in Tables S5 and S8. Hat: hours after treatment. 

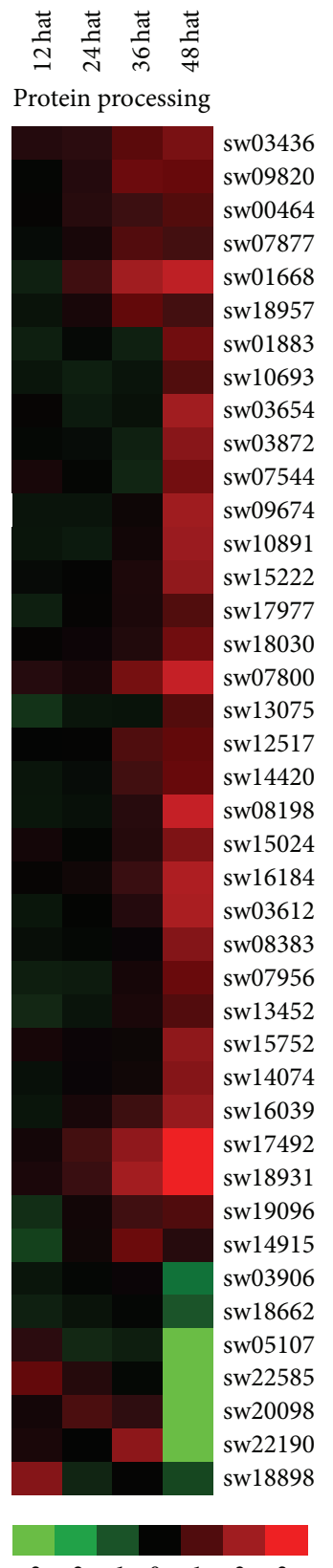

壳

Protein export

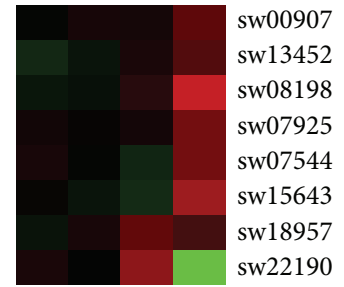

Ubiquitin-mediated proteolysis

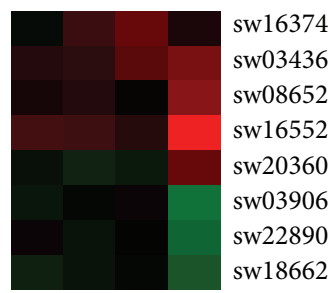

Peroxisome
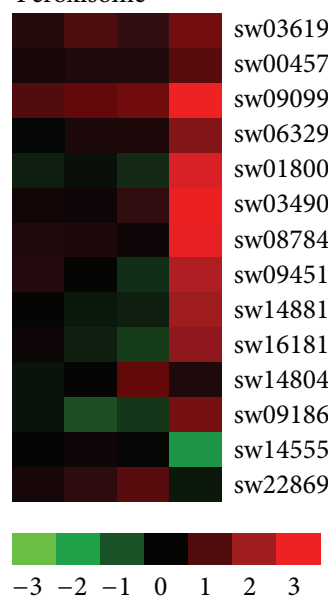

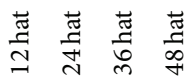

Proteasome

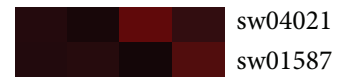

Lysosome

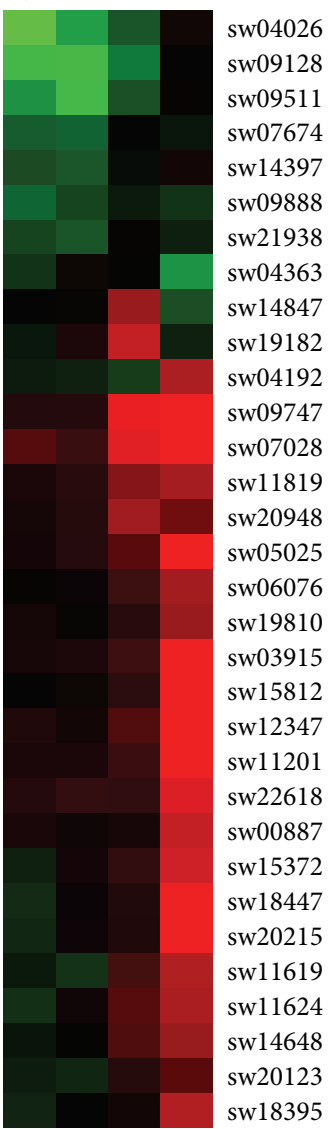

$\begin{array}{lllllll}-3 & -2 & -1 & 0 & 1 & 2 & 3\end{array}$

FIGURE 6: JHA-modulated pathways involved in protein synthesis and degradation. Hierarchical clustering of JHA-modulated genes involved in six pathways related to protein synthesis and degradation, including protein processing, protein export, ubiquitin-mediated proteolysis, proteasome, lysosome, and peroxisome. The probe ID, KEGG ID, and expression intensity of each JHA-modulated gene are listed in Tables S5 and S8. Hat: hours after treatment.

phosphatase F, starch phosphorylase, fatty acid synthase, fructose-1,6-bisphosphatase I, and protein phosphatase 1regulatory (inhibitor) subunit 3 ), and six genes for PI3KAkt signaling (heat shock protein $90 \mathrm{kDa}$ beta, laminin alpha, laminin beta, laminin gamma, protein phosphatase 2 regulatory subunit $A$, and thrombospondin). Importantly, real-time RT-PCR examination revealed that three key genes involved in the IIS pathway, PI3K, Akt, and $4 E-B P$, were also mediated by JHA application (Figure 7(b)). After JHA application, $P I 3 K$ and $A k t$ were upregulated, whereas $4 E-B P$ was downregulated in both the integument and the fat body.

Given that insulin signaling pathways are implicated in ecdysteroidogenesis in insects $[32,33]$ and larval molting is controlled by a $20 \mathrm{E}$ pulse, it was necessary to check whether genes involved in 20E-related pathways were also upregulated in JHA-induced silkworm superlarvae. As expected, the cytochrome p450 enzyme gene Cyp314a1 (Shade), which is responsible for converting ecdysone to active 20E, was 


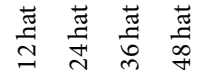

Insulin secretion

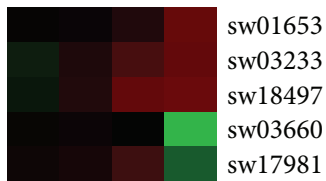

Insulin signaling

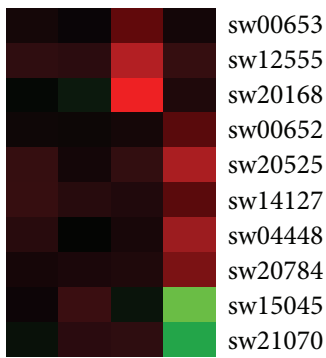

PI3K-Akt signaling

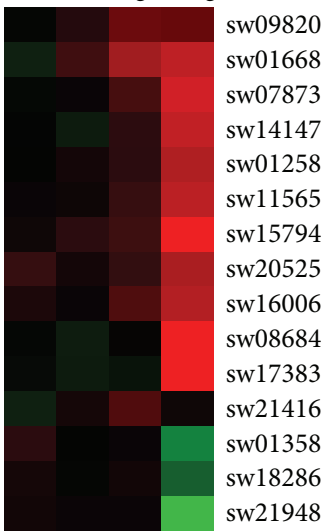

Ecdysteroidogenesisrelated Cyp314a1
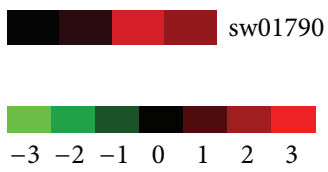

(a)
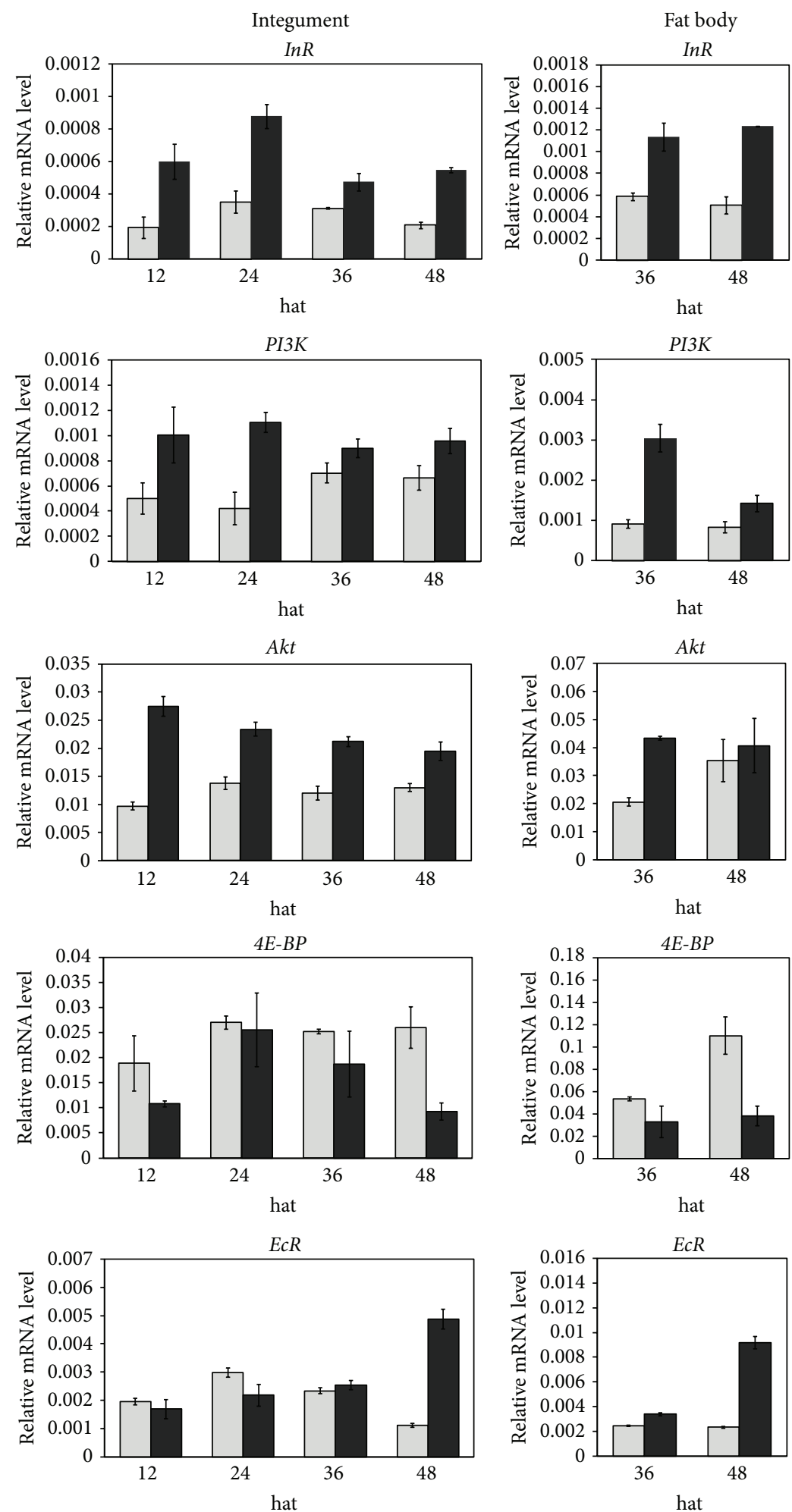

$$
\begin{aligned}
& \square \text { Acetone } \\
& \square \text { JHA }
\end{aligned}
$$

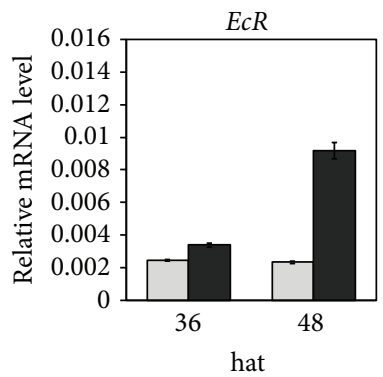

$\square$ Acetone

- JHA

FIGURE 7: The IIS pathway is modulated by JHA. (a) Hierarchical clustering of JHA-modulated genes related to the IIS pathway and 20E biosynthesis. The probe ID, KEGG ID, and expression intensity of each JHA-modulated gene are listed in Tables S5 and S8. Hat: hours after treatment. (b) Real-time RT-PCR analysis of JHA modulation of the expression levels of key regulators involved in the IIS pathway and 20E signaling. 
upregulated by JHA from 24 to 48 Hat (Figure 7(a); Table S8). The RT-PCR examination showed that the $20 \mathrm{E}$ receptor gene $E c R$ was also upregulated after JHA induction (Figure 7(b)).

\section{Discussion}

The larval stage in most holometabolic insects is characterized by nutrient-dependent growth and several times of larval molting. Insect larval growth and molting are mainly coordinated by two endocrine hormones, $\mathrm{JH}$ and 20E. JH maintains larval growth whereas nutrient-sensitive $20 \mathrm{E}$ signal triggers the initiation of larval molting. Therefore, larval growth and molting are excellent biological events for studying the crosstalk between $\mathrm{JH}$ and $20 \mathrm{E}$ in insects. Undoubtedly, insect larval growth must involve various metabolic processes. However, which metabolic processes are regulated by $\mathrm{JH}$ during larval growth and how $\mathrm{JH}$ contributes to $20 \mathrm{E}$ signaling have not been comprehensively examined. In this study, we used the model of JHA-induced silkworm superlarvae and microarray-based gene expression profiling to investigate the functions of $\mathrm{JH}$ in both the maintenance of metabolic homeostasis and the $20 \mathrm{E}$ based regulation of larval molting during silkworm larval stages.

Generally, the silkworm larva undergoes four larval molts and forms five larval instars. Previous studies reported that the application of the JHA methoprene to silkworm larvae at the beginning of the fourth larval instar will induce superlarvae with a supernumerary fifth molting [39]. To understand the mechanisms underlying the crosstalk between $\mathrm{JH}$ and $20 \mathrm{E}$ during the larval stage, we induced silkworm superlarvae by JHA application and observed that the onset of the fourth molt in superlarvae was approximately one day earlier than that in the control larvae treated with pure acetone (Figure 1). The JH-responsive gene $K r$ - $h 1$ also showed increased expression in two JH-targeted tissues of superlarvae, the integument and fat body, further confirming that the induction of silkworm superlarvae could be the consequence of JHA application.

To determine how silkworm larvae respond to JH during larval growth and molting, we performed a microarray-based analysis of gene expression changes after JHA application in the integument. A genomewide silkworm microarray has been previously used to profile gene expression in the silkworm [11, 40,41]. In addition, the identification of $\mathrm{JH}$-responsive genes has been performed in some specific cell lines or tissues as well as at different developmental stages in some insects, including the fruit fly (Drosophila melanogaster) $[8,9,12]$, yellow fever mosquito [7], red flour beetle [49], pine engraver beetle (Ips pini) [14], and silkworm $[11,13]$. Comparatively, some new and interesting findings were identified by our microarray analysis of the $\mathrm{JH}$ response in the silkworm integument.

Our studies first provide systematic insights into $\mathrm{JH}$ modulation on the programs controlling basic metabolism and protein processing during silkworm larval growth and molting. Comparative analysis showed that the expression levels of thousands of genes were significantly changed after
JHA application (Table S3). Interestingly, most of these JHAmodulated genes are involved in basic metabolic pathways (e.g., glycolysis, other carbohydrate metabolic programs, and lipid metabolism) and protein processing pathways. Furthermore, most JHA-modulated genes were upregulated after JHA application (Table S8), indicating that JHA application could promote most metabolic processes and protein processing programs. Previous reports have confirmed that a reduction in $\mathrm{JH}$ biosynthesis could decrease the levels of carbohydrate and lipid metabolism in the red flour beetle [24] and that $\mathrm{JH}$ application could upregulate both the mRNA levels and enzymatic activity of proteins related to glycolysis in the silkworm fat body [13]. Taken together, we propose that a JH-induced increase in basic metabolic activities may produce more nutritional supplies and further facilitate the growth of silkworm larvae.

An interesting observation of our study is that the mRNA level of trehalose-6-phosphate synthase (TPS), an enzyme controlling starch and sucrose metabolism, showed an obvious increase in the silkworm integument after JHA application, which is different from a previous finding showing no significant changes in TPS expression in the silkworm fat body after JHA application [24]. This finding raises the prospect that the JH-induced regulation of TPS transcription might be tissue specific in the silkworm. Intriguingly, our data also revealed that most of $\mathrm{JH}$-modulated genes that were involved in OXPHOS exhibited significantly reduced expression in the integument (Figure 4; Table S8). Generally, the OXPHOS pathway mediates energy metabolism and produces ATP via the oxidation of nutrients in the mitochondria of animal muscles $[50,51]$. Thus, the reduction of expression level of genes involved in the OXPHOS pathway in the silkworm integument before larval molting indicates that OXPHOS-mediated ATP production or requirements might be decreased after JHA application.

The IIS pathway acts as a nutrient sensor in the regulation of metabolic homeostasis and growth [52]. Previous evidences showed that the IIS pathway is stimulated by high glucose and regulates multiple metabolic pathways related to normal growth through various ways, for example, attenuating sugar levels, promoting glycolysis, and synthesizing fatty acids and proteins [53]. Surprisingly, our microarray data revealed that JHA application increased the expression of the insulin receptor gene $\operatorname{In} R$ in the integument and upregulated other genes involved in IIS-related pathways, including insulin secretion, insulin signaling, and the PI3K-Akt signaling pathway (Figure 7(a)). Real-time RT-PCR examination further confirmed that among several key regulators involved in the IIS pathway, InR, $P I 3 K$, and Akt showed an expected upregulation after JHA application, whereas $4 E-B P$ expression was decreased (Figure $7(\mathrm{~b})$ ), indicating that JHA application can increase the activity of the IIS pathway. Moreover, the fact that JHA application impaired the OXPHOS pathway also indicated an increased activity of the IIS pathway after JHA application because OXPHOS processes can be attenuated by abnormal nutritional signals (such as high insulin levels or a high-fat diet) in human skeletal muscle [54-57]. In combination with our observation of the JHA-induced modulation of carbohydrate metabolism, 
we speculate that $\mathrm{JH}$ might regulate carbohydrate metabolism to increase sugar levels in hemocytes, which could further activate the IIS pathway that is required for regulating glycolysis, lipid metabolism, and protein processing.

Insect larval molting is triggered by a pulse of $20 \mathrm{E}$ when the larvae grow to an appropriate body size under normal nutrient conditions $[58,59]$. Thus, JHA-induced premature larval molting indicates that JHA application might accelerate the emergence of $20 \mathrm{E}$ pulse in the fourth larval instar. In fact, $20 \mathrm{E}$ biosynthesis was previously reported to be catalyzed by several cytochrome P450 enzymes [60]. Our data showed that the expression of two 20E-related genes, Cyp314al (Shade), which encodes a P450 enzyme for transforming inactive ecdysone into active $20 \mathrm{E}$, and $E c R$, which encodes ecdysone receptor that is involved in $20 \mathrm{E}$ signaling, was significantly elevated following JHA application. This further confirmed that premature larval molting was likely resulted from the early emergence of 20E pulse after JHA application in the end of the fourth larval instar, which consequently contributed to shortening the developmental duration of the fourth larval instar and to subsequently reducing the body weight. In addition, we noted that compared to the early stages (12 and 24 Hat) after JHA application, a large number of genes showed more dramatic changes at the late stage of 48 Hat in expression levels, indicating that these JHA-modulated genes with dramatic expression changes may be just the indirect targets of $\mathrm{JH}$, but the targets of $20 \mathrm{E}$ to a great extent. Furthermore, it has been known that insect metamorphosis with larvalpupal transition is also determined by a critical weight that is related to body size [61]. Given that the body weight of the JHA-induced superlarvae in the fifth larval instar was also reduced, we suggested that in the fifth larval instar, JHAinduced silkworm superlarvae did not reach to critical weight as same with that in the control, so it should experience an additional larval molting, which may be the same as the fourth instar of the control. Moreover, previous reports have demonstrated that $20 \mathrm{E}$ biosynthesis is also positively mediated by insulin signaling $[32,34,62]$. Together with our observation that genes involved in the IIS pathway were significantly upregulated after JHA application, we therefore suggest that $\mathrm{JH}$ may regulate $20 \mathrm{E}$ signaling by mediating basic metabolic activities and the nutrient-dependent IIS pathway. Further in vivo and in vitro experiments will be needed to test this hypothesis.

\section{Conclusion}

In this study, we profiled the genomewide alteration of gene expression in the integument of silkworm larvae after JHA application and found the cues about $\mathrm{JH}$ regulation on metabolic homeostasis, IIS pathway, and 20E signaling. Further studies should be conducted and may be helpful for deciphering the mechanism underlying the interaction between $\mathrm{JH}$ and $20 \mathrm{E}$ during silkworm larval growth.

\section{Abbreviations}

$\mathrm{JH}$ : Juvenile hormone

JHA: Juvenile hormone analogue
20E: 20-Hydroxyecdysone

IIS: Insulin/insulin-like growth factor signaling

Hat: Hours after treatment

OXPHOS: Oxidative phosphorylation.

\section{Conflict of Interests}

The authors declare that there is no conflict of interests regarding the publication of this paper.

\section{Authors' Contribution}

Daojun Cheng and Jian Peng contributed equally to this work.

\section{Acknowledgments}

The authors thank the reviewers for their comments and suggestions. This work was supported by Grants from the National Basic Research Program of China (no. 2012CB114600), the National Hi-Tech Research and Development Program of China (no. 2011AA100306), the National Natural Science Foundation of China (no. 31172267 and no. 31272503), and the Municipal Natural Science Foundation of Chongqing (no. cstc2012jjA80023).

\section{References}

[1] L. M. Riddiford, "Cellular and molecular actions of juvenile hormone. I. General considerations and premetamorphic actions," Advances in Insect Physiology, vol. 24, pp. 213-274, 1994.

[2] G. R. Wyatt and K. G. Davey, "Cellular and molecular actions of juvenile hormone. II. Roles of juvenile hormone in adult insects," Advances in Insect Physiology, vol. 26, pp. 1-155, 1996.

[3] S. Niimi and S. Sakurai, "Development changes in juvenile hormone and juvenile hormone acid titers in the hemolymph and in-vitro juvenile hormone synthesis by corpora allata of the silkworm, Bombyx mori," Journal of Insect Physiology, vol. 43, no. 9, pp. 875-884, 1997.

[4] L. M. Riddiford, "Juvenile hormone: the status of its "status quo" action," Archives of Insect Biochemistry and Physiology, vol. 32, no. 3-4, pp. 271-286, 1996.

[5] E. B. Dubrovsky, "Hormonal cross talk in insect development," Trends in Endocrinology and Metabolism, vol. 16, no. 1, pp. 6-11, 2005.

[6] L. M. Riddiford, "How does juvenile hormone control insect metamorphosis and reproduction?" General and Comparative Endocrinology, vol. 179, no. 3, pp. 477-484, 2012.

[7] Z. Zou, T. T. Saha, S. Roy et al., "Juvenile hormone and its receptor, methoprene-tolerant, control the dynamics of mosquito gene expression," Proceedings of the National Academy of Sciences of the United States of America, vol. 110, no. 24, pp. E2173-E2181, 2013.

[8] D. K. Willis, J. Wang, J. R. Lindholm, A. Orth, and W. G. Goodman, "Microarray analysis of juvenile hormone response in Drosophila melanogaster S2 cells," Journal of Insect Science, vol. 10, no. 66, pp. 1-14, 2010.

[9] Y. Li, Z. Zhang, G. E. Robinson, and S. R. Palli, "Identification and characterization of a juvenile hormone response element 
and its binding proteins," The Journal of Biological Chemistry, vol. 282, no. 52, pp. 37605-37617, 2007.

[10] M. Li, E. A. Mead, and J. S. Zhu, "Heterodimer of two bHLHPAS proteins mediates juvenile hormone-induced gene expression," Proceedings of the National Academy of Sciences of the United States of America, vol. 108, no. 2, pp. 638-643, 2011.

[11] L. Tian, E. Guo, Y. Diao et al., "Genome-wide regulation of innate immunity by juvenile hormone and 20-hydroxyecdysone in the Bombyx fat body," BMC Genomics, vol.11, no. 1, article 549, 2010.

[12] R. B. Beckstead, G. Lam, and C. S. Thummel, "Specific transcriptional responses to juvenile hormone and ecdysone in Drosophila," Insect Biochemistry and Molecular Biology, vol. 37, no. 6, pp. 570-578, 2007.

[13] L. Tian, E. Guo, S. Wang et al., "Developmental regulation of glycolysis by 20 -hydroxyecdysone and juvenile hormone in fat body tissues of the silkworm, Bombyx mori," Journal of Molecular Cell Biology, vol. 2, no. 5, pp. 255-263, 2010.

[14] C. I. Keeling, J. C. Bearfield, S. Young, G. J. Blomquist, and C. Tittiger, "Effects of juvenile hormone on gene expression in the pheromone-producing midgut of the pine engraver beetle, Ips pini," Insect Molecular Biology, vol. 15, no. 2, pp. 207-216, 2006.

[15] C. Minakuchi, T. Namiki, and T. Shinoda, "Krüppel homolog 1 , an early juvenile hormone-response gene downstream of Methoprene-tolerant, mediates its anti-metamorphic action in the red flour beetle Tribolium castaneum," Developmental Biology, vol. 325, no. 2, pp. 341-350, 2009.

[16] C. Minakuchi, X. Zhou, and L. M. Riddiford, "Krüppel homolog1 (Kr-h1) mediates juvenile hormone action during metamorphosis of Drosophila melanogaster," Mechanisms of Development, vol. 125, no. 1-2, pp. 91-105, 2008.

[17] T. Kayukawa, C. Minakuchi, T. Namiki et al., "Transcriptional regulation of juvenile hormone-mediated induction of Krüppel homolog 1, a repressor of insect metamorphosis," Proceedings of the National Academy of Sciences of the United States of America, vol. 109, no. 29, pp. 11729-11734, 2012.

[18] J. Lozano and X. Belles, "Conserved repressive function of Krüppel homolog 1 on insect metamorphosis in hemimetabolous and holometabolous species," Scientific Reports, vol. 1, article $163,2011$.

[19] M. Jindra, S. R. Palli, and L. M. Riddiford, "The juvenile hormone signaling pathway in insect development," Annual Review of Entomology, vol. 58, pp. 181-204, 2013.

[20] D. Stanojevic, T. Hoey, and M. Levine, "Sequence-specific DNAbinding activities of the gap proteins encoded by hunchback and Krüppel in Drosophila," Nature, vol. 341, no. 6240, pp. 331-335, 1989.

[21] T. Kayukawa, K. Tateishi, and T. Shinoda, "Establishment of a versatile cell line for juvenile hormone signaling analysis in Tribolium castaneum," Scientific Reports, vol. 3, Article ID 1570, 2013.

[22] W. Chefurka, "Sesquiterpene juvenile hormones: novel uncouplers of oxidative phosphorylation," Biochemical and Biophysical Research Communications, vol. 83, no. 2, pp. 571-578, 1978.

[23] A. A. Baumann, J. B. Benoit, V. Michalkova et al., "Juvenile hormone and insulin suppress lipolysis between periods of lactation during tsetse fly pregnancy," Molecular and Cellular Endocrinology, vol. 372, no. 1-2, pp. 30-41, 2013.

[24] J. Xu, Z. Sheng, and S. R. Palli, "Juvenile hormone and insulin regulate trehalose homeostasis in the red flour beetle, Tribolium castaneum," PLoS Genetics, vol. 9, no. 6, article e1003535, 2013.
[25] Q. Wu and M. R. Brown, "Signaling and function of insulin-like peptides in insects," Annual Review of Entomology, vol. 51, pp. 124, 2006.

[26] M. Perez-Hedo, C. Rivera-Perez, and F. G. Noriega, “The insulin/TOR signal transduction pathway is involved in the nutritional regulation of juvenile hormone synthesis in Aedes aegypti," Insect Biochemistry and Molecular Biology, vol. 43, no. 6, pp. 495-500, 2013.

[27] M. P. Tu, C. M. Yin, and M. Tatar, "Mutations in insulin signaling pathway alter juvenile hormone synthesis in Drosophila melanogaster," General and Comparative Endocrinology, vol. 142, no. 3, pp. 347-356, 2005.

[28] T. Koyama, M. O. Syropyatova, and L. M. Riddiford, "Insulin/IGF signaling regulates the change in commitment in imaginal discs and primordia by overriding the effect of juvenile hormone," Developmental Biology, vol. 324, no. 2, pp. 258-265, 2008.

[29] E. E. Guo, Q. Y. He, S. M. Liu et al., "MET is required for the maximal action of 20-hydroxyecdysone during Bombyx metamorphosis," PLoS ONE, vol. 7, no. 12, article e53256, 2012.

[30] Y. Liu, Z. Sheng, H. H. Liu et al., "Juvenile hormone counteracts the bHLH-PAS transcription factors MET and GCE to prevent caspase-dependent programmed cell death in Drosophila," Development, vol. 136, no. 12, pp. 2015-2025, 2009.

[31] B. Konopova and M. Jindra, "Broad-complex acts downstream of Met in juvenile hormone signaling to coordinate primitive holometabolan metamorphosis," Development, vol. 135, no. 3, pp. 559-568, 2008.

[32] S.-H. Gu, J.-L. Lin, P.-L. Lin, and C.-H. Chen, "Insulin stimulates ecdysteroidogenesis by prothoracic glands in the silkworm, Bombyx mori," Insect Biochemistry and Molecular Biology, vol. 39, no. 3, pp. 171-179, 2009.

[33] S. Layalle, N. Arquier, and P. Leopold, "The TOR pathway couples nutrition and developmental timing in Drosophila," Developmental Cell, vol. 15, no. 4, pp. 568-577, 2008.

[34] S.-H. Gu, S.-C. Young, J.-L. Lin, and P.-L. Lin, "Involvement of PI3K/Akt signaling in PTTH-stimulated ecdysteroidogenesis by prothoracic glands of the silkworm, Bombyx mori," Insect Biochemistry and Molecular Biology, vol. 41, no. 3, pp. 197-202, 2011.

[35] A. Mizoguchi, "Effects of juvenile hormone on the secretion of prothoracicotropic hormone in the last- and penultimateinstar larvae of the silkworm Bombyx mori," Journal of Insect Physiology, vol. 47, no. 7, pp. 767-775, 2001.

[36] A. Tan, H. Tanaka, T. Tamura, and T. Shiotsuki, "Precocious metamorphosis in transgenic silkworms overexpressing juvenile hormone esterase," Proceedings of the National Academy of Sciences of the United States of America, vol. 102, no. 33, pp. 11751-11756, 2005.

[37] S.-H. Gu and W.-H. Tsai, "Decreased JH biosynthesis is related to precocious metamorphosis in recessive trimolter $(r t)$ mutants of the silkworm, Bombyx mori," Archives of Insect Biochemistry and Physiology, vol. 79, no. 4-5, pp. 235-246, 2012.

[38] T. Daimon, T. Kozaki, R. Niwa et al., "Precocious metamorphosis in the juvenile hormone-deficient mutant of the silkworm, Bombyx mori," PLoS Genetics, vol. 8, no. 3, article e1002486, 2012.

[39] K. Kadonookuda, Z. Kajiura, and O. Yamashita, "Induction of perfect superlarvae by the application of juvenile hormone analogue to starved larvae of the silkworm, Bombyx mori," Journal of Insect Physiology, vol. 32, no. 12, pp. 1065-1073, 1986. 
[40] L. Huang, T. Cheng, P. Xu, D. Cheng, T. Fang, and Q. Xia, “A genome-wide survey for host response of silkworm, Bombyx mori during pathogen Bacillus bombyseptieus infection," PLoS ONE, vol. 4, no. 12, p. e8098, 2009.

[41] Q. Xia, D. Cheng, J. Duan et al., "Microarray-based gene expression profiles in multiple tissues of the domesticated silkworm, Bombyx mori," Genome Biology, vol. 8, no. 8, article R162, 2007.

[42] K. Hartfelder, "Insect juvenile hormone: from "status quo" to high society," Brazilian Journal of Medical and Biological Research, vol. 33, no. 2, pp. 157-177, 2000.

[43] Q. Xia, S. Li, and Q. Feng, "Advances in silkworm studies accelerated by the genome sequencing of Bombyx mori," Annual Review of Entomology, vol. 59, pp. 513-536, 2014.

[44] M. B. Eisen, P. T. Spellman, P. O. Brown, and D. Botstein, "Cluster analysis and display of genome-wide expression patterns," Proceedings of the National Academy of Sciences of the United States of America, vol. 95, no. 25, pp. 14863-14868, 1998.

[45] M. Reich, K. Ohm, M. Angelo, P. Tamayo, and J. P. Mesirov, "GeneCluster 2.0: an advanced toolset for bioarray analysis," Bioinformatics, vol. 20, no. 11, pp. 1797-1798, 2004.

[46] J. Ye, L. Fang, H. Zheng et al., "WEGO: a web tool for plotting GO annotations," Nucleic Acids Research, vol. 34, supplement 2, pp. W293-W297, 2006.

[47] Y. Moriya, M. Itoh, S. Okuda, A. C. Yoshizawa, and M. Kanehisa, "KAAS: an automatic genome annotation and pathway reconstruction server," Nucleic acids research, vol. 35, supplement 2, pp. W182-W185, 2007.

[48] K. Cartharius, K. Frech, K. Grote et al., "MatInspector and beyond: promoter analysis based on transcription factor binding sites," Bioinformatics, vol. 21, no. 13, pp. 2933-2942, 2005.

[49] R. Parthasarathy and S. R. Palli, "Molecular analysis of juvenile hormone analog action in controlling the metamorphosis of the red flour beetle, Tribolium castaneum," Archives of Insect Biochemistry and Physiology, vol. 70, no. 1, pp. 57-70, 2009.

[50] G. N. Ling, "Oxidative phosphorylation and mitochondrial physiology: a critical review of chemiosmotic theory, and reinterpretation by the association-induction hypothesis," Physiological Chemistry and Physics, vol. 13, no. 1, pp. 29-96, 1981.

[51] A. E. Senior, "ATP synthesis by oxidative phosphorylation," Physiological Reviews, vol. 68, no. 1, pp. 177-231, 1988.

[52] C. J. Kenyon, “The genetics of ageing," Nature, vol. 464, no. 7288, pp. 504-512, 2010.

[53] G. Dimitriadis, P. Mitron, V. Lambadiari, E. Maratou, and S. A. Raptis, "Insulin effects in muscle and adipose tissue," Diabetes Research and Clinical Practice, vol. 93, supplement 1, pp. S52S59, 2011.

[54] A. H. Olsson, B. T. Yang, E. Hall et al., "Decreased expression of genes involved in oxidative phosphorylation in human pancreatic islets from patients with type 2 diabetes," European Journal of Endocrinology, vol. 165, no. 4, pp. 589-595, 2011.

[55] V. K. Mootha, C. M. Lindgren, K.-F. Eriksson et al., "PGC$1 \alpha$-responsive genes involved in oxidative phosphorylation are coordinately downregulated in human diabetes," Nature Genetics, vol. 34, no. 3, pp. 267-273, 2003.

[56] C. S. Stump, K. R. Short, M. L. Bigelow, J. M. Schimke, and K. S. Nair, "Effect of insulin on human skeletal muscle mitochondrial ATP production, protein synthesis, and mRNA transcripts," Proceedings of the National Academy of Sciences of the United States of America, vol. 100, no. 13, pp. 7996-8001, 2003.
[57] M. Wang, X. C. Wang, Z. Y. Zhang, B. Mou, and R. M. Hu, "Impaired mitochondrial oxidative phosphorylation in multiple insulin-sensitive tissues of humans with type 2 diabetes mellitus," The Journal of International Medical Research, vol. 38, no. 3, pp. 769-781, 2010.

[58] A. Telang, L. Frame, and M. R. Brown, "Larval feeding duration affects ecdysteroid levels and nutritional reserves regulating pupal commitment in the yellow fever mosquito Aedes aegypti (Diptera: Culicidae)," The Journal of Experimental Biology, vol. 210, no. 5, pp. 854-864, 2007.

[59] L. M. Riddiford, K. Hiruma, X. F. Zhou, and C. A. Nelson, "Insights into the molecular basis of the hormonal control of molting and metamorphosis from Manduca sexta and Drosophila melanogaster," Insect Biochemistry and Molecular Biology, vol. 33, no. 12, pp. 1327-1338, 2003.

[60] L. I. Gilbert, "Halloween genes encode P450 enzymes that mediate steroid hormone biosynthesis in Drosophila melanogaster," Molecular and Cellular Endocrinology, vol. 215, no. 1-2, pp. 1-10, 2004.

[61] E. T. Danielsen, M. E. Moeller, and K. F. Rewitz, "Nutrient signaling and developmental timing of maturation," Current Topics in Developmental Biology, vol. 105, pp. 37-67, 2013.

[62] M. A. Riehle and M. R. Brown, "Insulin stimulates ecdysteroid production through a conserved signaling cascade in the mosquito Aedes aegypti," Insect Biochemistry and Molecular Biology, vol. 29, no. 10, pp. 855-860, 1999. 

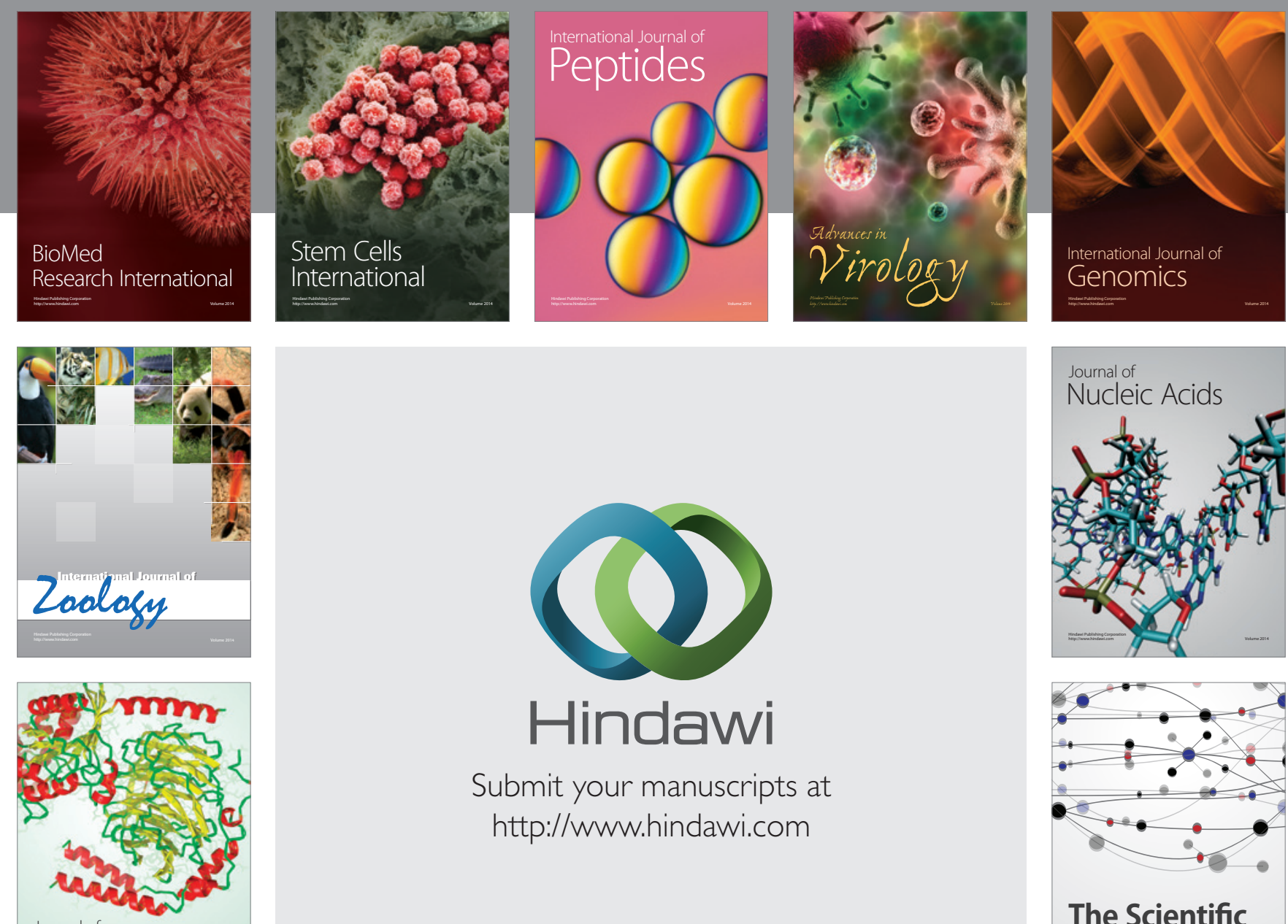

Submit your manuscripts at

http://www.hindawi.com

Journal of
Signal Transduction
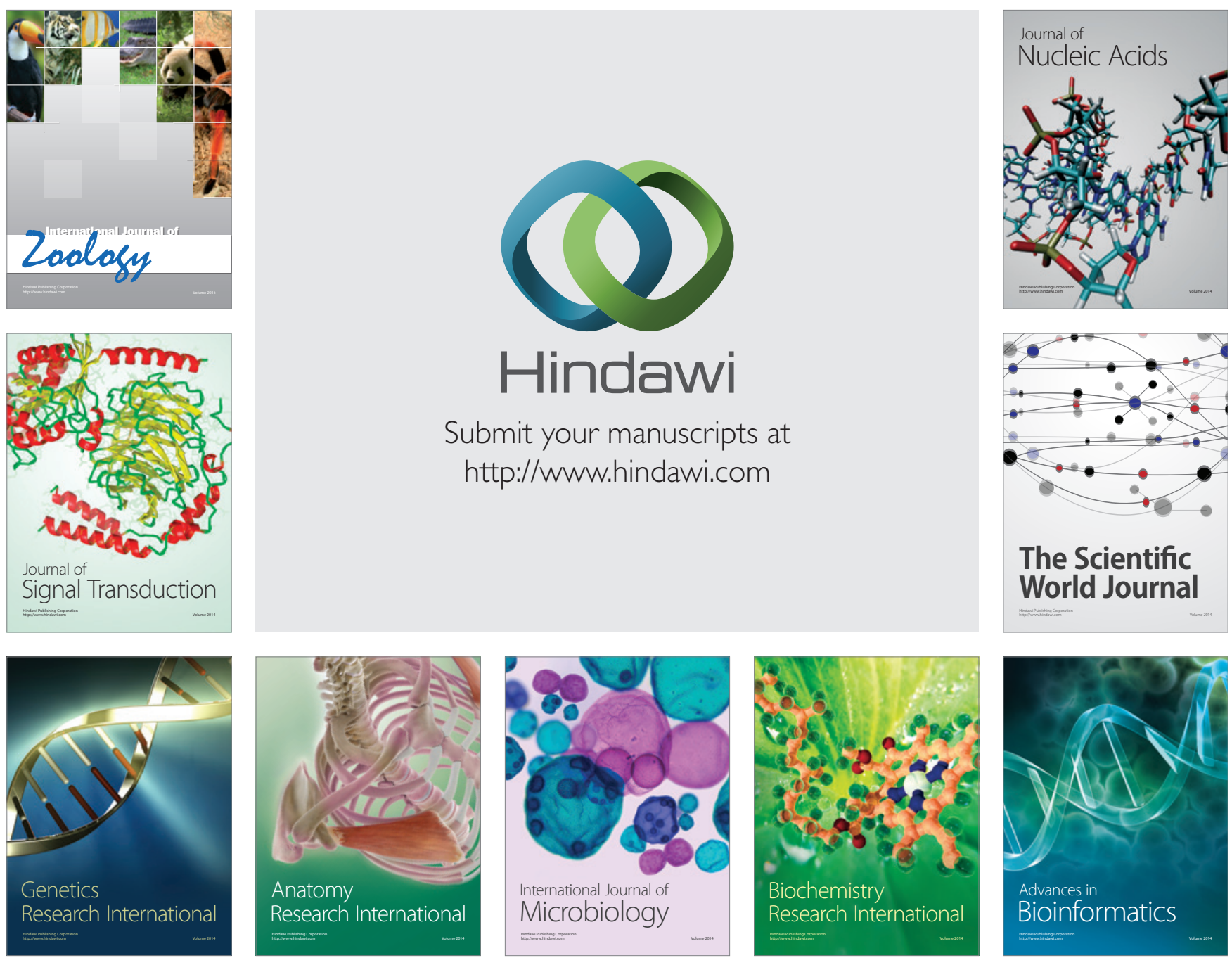

The Scientific World Journal
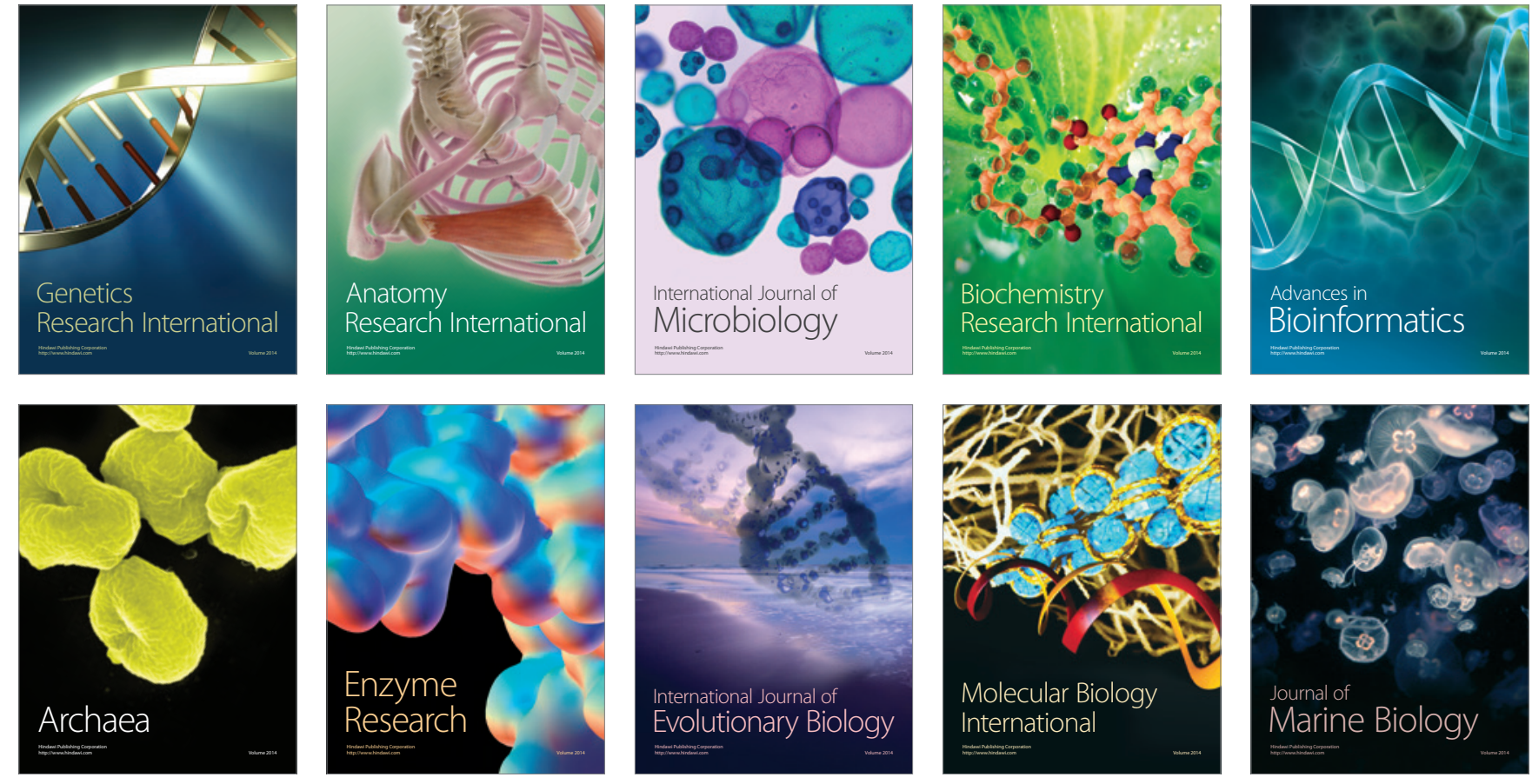